\title{
Parametrized tests of post-Newtonian theory using Advanced LIGO and Einstein Telescope
}

\author{
Chandra Kant Mishra, ${ }^{1,2, *}$ K. G. Arun, ${ }^{3, \dagger}$ Bala R. Iyer, ${ }^{1, \ddagger}$ and B. S. Sathyaprakash ${ }^{4, \S}$ \\ ${ }^{1}$ Raman Research Institute, Bangalore, 560 080, India \\ ${ }^{2}$ Department of Physics, Indian Institute of Science, Bangalore, 560 012, India \\ ${ }^{3}$ McDonnell Center for the Space Sciences, Department of Physics, Washington University, St. Louis, Missouri 63130, USA \\ ${ }^{4}$ School of Physics and Astronomy, Cardiff University, 5, The Parade, Cardiff, United Kingdom, CF24 $3 Y B$
}

(Received 4 May 2010; published 7 September 2010)

\begin{abstract}
General relativity has very specific predictions for the gravitational waveforms from inspiralling compact binaries obtained using the post-Newtonian (PN) approximation. We investigate the extent to which the measurement of the PN coefficients, possible with the second generation gravitational-wave detectors such as the Advanced Laser Interferometer Gravitational-Wave Observatory (LIGO) and the third generation gravitational-wave detectors such as the Einstein Telescope (ET), could be used to test post-Newtonian theory and to put bounds on a subclass of parametrized-post-Einstein theories which differ from general relativity in a parametrized sense. We demonstrate this possibility by employing the best inspiralling waveform model for nonspinning compact binaries which is 3.5PN accurate in phase and $3 \mathrm{PN}$ in amplitude. Within the class of theories considered, Advanced LIGO can test the theory at 1.5PN and thus the leading tail term. Future observations of stellar mass black hole binaries by ET can test the consistency between the various PN coefficients in the gravitational-wave phasing over the mass range of $11-44 M_{\odot}$. The choice of the lower frequency cutoff is important for testing post-Newtonian theory using the ET. The bias in the test arising from the assumption of nonspinning binaries is indicated.
\end{abstract}

DOI: 10.1103/PhysRevD.82.064010

\section{INTRODUCTION}

General relativity is tested to unprecedented accuracies in the weak-field and strong-field regimes (see Ref. [1] for a review). From a theoretical perspective, tests of this nature were possible due to physically motivated but structurally simple parametrizations of the observable quantities which could have different values in different theories of gravity. In the weak-field regime, solar system bounds were largely assisted by the parametrized post-Newtonian (PPN) framework (see, for example, Ref. [2]). PPN formalism parametrizes the deviation of a general metric theory of gravity (with symmetric metric) from the Newtonian theory in the weak-field limit in terms of 10 free parameters up to order $v / c$, where $v$ is the characteristic velocity of the object. General relativity (GR) is a special case of this class with specific values of these parameters.

Binary pulsar tests, which dealt with stronger gravitational fields involving compact objects but typical velocities of order $v \sim 10^{-3} c$, probed GR in the strong-field radiative regime. The binary pulsar tests were performed effectively with the use of the parametrized post-Keplerian (PPK) [3-6] formulation of the pulsar timing formula. The timing formula can be expressed as a function of Keplerian and post-Keplerian parameters, each one of which is a function of the component masses of the binary. A measurement of two of these parameters enabled the

\footnotetext{
*chandra@rri.res.in

†arun@physics.wustl.edu

bri@rri.res.in

${ }^{\S}$ B.Sathyaprakash@astro.cf.ac.uk
}

PACS numbers: 04.30.Db, 04.25.Nx, 04.80.Nn, 95.55.Ym

determination of the individual masses. The measurement of a third parameter would constitute a test of the theory, by requiring a consistency of the component masses in the $m_{1}-m_{2}$ plane. Depending on the number of these PPK parameters that can be measured from the timing data of a binary pulsar, it enables many tests of GR (measuring $n$ parameters allow $n-2$ tests). Binary pulsar observations also confirmed the quadrupole formula for the generation of gravitational waves.

\section{A. Gravitational waves and tests of GR}

The detection of gravitational waves (GWs) would be the first direct test of the consistency of gravitation with the principles of special relativity and would probe general relativity beyond the quadrupole formula [7]. A subsequent detailed study of the properties of GW would next allow one to assess the validity of GR in the strong-field radiative regime. A prominent class of $\mathrm{GW}$ sources is compact binaries: neutron stars (NS) and/or black holes (BH) moving in circular orbit with velocities $v \sim 0.2 c$. Within GR, using different analytical and numerical schemes, gravitational waveforms from these systems can be computed with very high accuracy [8]. Availability of such high-accuracy waveforms will allow the application of matched filtering techniques to search for these signals in the data from the GW interferometers such as LIGO [9] and Virgo [10].

Beyond the detection of GWs, one would like to know whether one can perform tests of GR with the detected signals. Despite the use of GR waveforms in matched filtering (which essentially assumes that GR is the correct theory of gravity), several authors have argued that GW 
observations can be used to test GR and put bounds on various parameters in alternative theories of gravity. One of the first proposals toward testing nonlinear aspects of GR using GWs was due to Blanchet and Sathyaprakash, who discussed the possibility of measuring the "tail" effect in the GW phasing formula $[11,12]$. Ryan proposed a method to measure various multipole moments of a binary system [13] from the Laser Interferometer Space Antenna (LISA) observations of extreme mass ratio inspirals. Will obtained the additional contributions to the GW phasing formula in Brans-Dicke theories [14] and massive graviton theories [15] as a one-parameter deviation from GR and discussed the bounds possible on these corresponding parameters from GW observations. These ideas were elaborated on in greater detail in a series of papers [16-21], studying how various physical effects in the binary affect the bounds. It is worth noting that these bounds possible on massive graviton theories will be complementary to those which are obtained by binary pulsar observations (see e.g. [22]).

The basic idea of our proposal can be viewed as a generalization of some of the existing proposals to test specific theories of gravity like Brans-Dicke or massive graviton theories. General relativity and any of its parametrized variants have different predictions for the PN coefficients $\psi_{i}$ in the phasing formula (for details see Sec. II C). Hence the accuracies with which the PN coefficients of GR can be measured translates into bounds on the values of these coefficients in any other theory. This leads to the question of how well can these coefficients be measured?

One way to answer this question is to rephrase it as a parameter estimation problem and measure each of the PN coefficients, treating them all as independent of one another. Recall that, for nonspinning binaries, each one of them is a function of only the two component masses and hence just two parameters are enough to describe the phasing formula up to any PN order. Hence, if we want to treat each one of them ( 8 in all for the restricted waveform (RWF) at 3.5PN order) independently, there will be large correlations among the parameters. Our earlier work [23] has shown that this method works well only for a narrow range of masses for which the signal-to-noise ratio (SNR) is high enough to discriminate these terms. The masses are typically of a million solar mass and hence detectable by the LISA [23,24].

Since the high correlations among the PN parameters are responsible for the ineffectiveness of the above test, we explored other possibilities which, though less generic, are viable, interesting and capture the essential features of the test. One possibility is to use a smaller set of parameters comprised of the PN coefficient to be tested together with any two of the remaining PN coefficients chosen as basic variables (to reexpress and parametrize the rest of the PN coefficients) [25].

While all these were based on inspiral waveforms, there are proposed tests based on merger and ringdown waveforms of the binary as well by measuring very accurately the various ringdown modes of the GW spectrum [26-28]. Keppel and Ajith [29] revisited the bounds on massive graviton theories including the merger and ringdown contributions. Alexander et al. pointed out that the GW observations can be used as a probe of effective quantum gravity which predict amplitude birefringence of the spacetime for the propagation of the GW signals [30]. Molina et al. investigated the possible imprints of Chern-Simon theory of gravity in the GW ringdown signals and its detectability with GW interferometers [31]. In brief, GW measurements can lead to interesting tests of various strong-field aspects of gravity.

Recently, Yunes and Pretorius discussed a generalized framework called the parametrized post-Einsteinian ( $\mathrm{ppE}$ ) framework to describe various fundamental biases in theoretical modeling of GW and express them in a parametrized manner [32]. They used the existing knowledge about various alternative theories of gravity such as BransDicke, massive graviton theories, and Chern-Simon theory to write down a generic Fourier domain gravitational waveform, which is parametrized in terms of a set of amplitude and phase variables. They also considered the contribution from merger and ringdown phases of the binary evolution beyond the inspiral. This parametrization in the inspiral regime can be considered as a generalization of our earlier proposal in Ref. [23] but including the possibility that amplitude of the waveform may also, in general, be different in an alternative theory.

In the present work, our aim is to set up a general parametrization of the gravitational-wave signal in a subclass of ppE theories that will enable tests of GR in the radiative regime from $\mathrm{GW}$ observations, similar to the PPN and PPK formalisms mentioned earlier. This is an extension of our previous work [25] using more complete inspiral waveforms, called full waveforms (see Sec. IC for a detailed discussion). From the ppE perspective, the model we have presented in this paper would correspond to the case where there are no amplitude deviations and only one phasing coefficient (corresponding to the test parameter) different from GR. In the future the more general $\mathrm{ppE}$ class of meta models must be investigated to provide more generic results than those obtained from the subclass we deal with in this paper.

\section{B. Choice of PN parametrization}

The suggestion to use a smaller set of parameters comprised of the PN coefficient to be tested together with any two of the remaining PN coefficients selected as basic variables (to reexpress and parametrize the rest of the PN coefficients) [25] immediately raises the following question. Which two parameters should be chosen as the basic variables? The most natural choice is the two lowest-order OPN and 1PN coefficients since they are measured most accurately. Furthermore, within GR at higher PN order there are spin-orbit and spin-spin terms etc., so that not 
only the choice of higher-order coefficients as basic parameters appears less convenient but the use of the lowestorder PN coefficients as basic variables may be expected to reduce systematic effects due to spins. Once the basic variables are decided, the test parameter can be any of the higher PN order coefficients, chosen one at a time.

The weakness of this version of the test vis-à-vis, the version where all $\mathrm{PN}$ parameters are treated as independent parameters, may be worrying at first. Unlike in the latter version where the discrepant PN order will be explicit in the test, in the former version the failure of GR at a particular PN order may not necessarily imply the corresponding PN term to be different from GR. However, we believe in the robustness of the test itself due to the following chain of plausibility arguments: It is not unreasonable to assume that if an alternative theory of gravitation is consistent with GR at some PN order it would be normally consistent with GR at lower PN orders but it may disagree with GR at some higher PN order. In view of this argument, if one is testing a particular order PN coefficient then parametrizing the lower-order PN coefficients by the basic PN coefficients (at $\mathrm{OPN}$ and 1PN orders) is reasonable.

What then do we hope to achieve by our choice of expressing the PN coefficients of order higher than the tested PN coefficient by the basic PN coefficients? To answer this question, we make the reasonable assumption that if a theory differs from GR at some PN order, it is likely to differ from GR at higher PN orders too. Thus by parametrizing PN coefficients of orders higher than the tested PN coefficient by the basic PN coefficients, we naturally take into account effects in GR coming from higher-order PN terms and reduce the corresponding systematic errors arising from the higher-order PN coefficients in the estimation of the test parameter. In consequence, only departures of the correct theory of gravity from GR would remain and contribute dominantly to the estimate of the PN parameter tested in the analysis. To investigate this question explicitly, we computed the error in the estimation of a particular PN coefficient both at the 3.5PN accuracy and by truncating the expansion at the PN order of the test parameter. As expected, the two choices gave different results, with the full phasing yielding a more accurate estimation of parameters (for more details, see Sec. III B 3). Given the possibly small differences we are trying to explore any reduction in systematic errors is indeed to be taken advantage of.

It is interesting to note that, as pointed out in Ref. [33], the errors in the various PN coefficients we quote here can be translated into measurement of three- and four-graviton vertices. Keeping these caveats in view, let us consider some hypothetical theory of gravity which shows deviation from GR starting from $2 \mathrm{PN}$ order. ${ }^{1}$ In our proposed test,

\footnotetext{
${ }^{1}$ There are theories which show deviation from GR starting from 2PN order. See, for example, Ref. [34] which considers one such example, though for spinning BHs.
}

this deviation would not show up when $\psi_{3}$ is used as test parameter, as in this case the deviations are only from the fact that the functional dependences of higher-order phasing coefficients on $\psi_{0}$ and $\psi_{2}$ are not the same as in GR. This seems less important than a lower PN order test parameter itself deviating from its GR value. On the other hand, when $\psi_{4}$ or higher PN order phasing coefficients are used as test parameters, this deviation should be evident. Thus, proceeding systematically to higher PN orders, one can ascertain the PN order where the new theory begins to deviate from GR.

\section{Scope of the current work and a summary of results}

In this work we revisit the problem in the context of the second generation ground-based GW interferometer such as Advanced LIGO and a third generation ground-based GW interferometer called Einstein Telescope (ET) that is currently under design study in Europe. Since ET is envisaged to have far better low-frequency sensitivity than Advanced LIGO (a lower frequency cutoff of about $1-5 \mathrm{~Hz}$ ), one of the aims of the present investigation is to evaluate the possible gains in going from a lower cutoff of 10 to $1 \mathrm{~Hz}$. A further new ingredient in the present version of the above test is that we use not just the 3.5PN RWF but also the amplitude-corrected full waveforms (FWF) which are 3PN accurate in amplitude (thus having seven harmonics other than just the leading quadrupolar one) and 3.5PN accurate in phase. For nonspinning binaries, the amplitude corrections are functions of the two masses and the inclination angle of the binary. The amplitude corrections at every PN order bring new dependences on the binary masses and hence could improve the estimation of the phasing coefficients. With our previous insight in the weakening of the test due to the use of more parameters [23], in the first instance, we make the reasonable assumption that since matched filtering procedure is more sensitive to the phase rather than amplitude, one can skip parametrizing the amplitude independently in the present work. Thus the deviations in the amplitude corrections of the waveform are not independently parametrized even though they have been taken account of in the present work to reduce systematic effects. We rewrite the mass dependences in the PN amplitude terms in terms of $\psi_{0}$ and $\psi_{2}$, just as in the case of the phase terms.

An obvious limitation in regard to the present analysis concerns the generality of the parametrization that we employ. Indeed, in the strong-field regime, alternative theories may so qualitatively deviate from GR that the structure of the waveform used here for the parametrization may not be generic enough to capture those features and one may need to redo the present analysis within a more general class of models like the ppE framework. It is worth mentioning that at present very accurate high PN order GW phasing results are available only for GR. Most results for alternative theories of gravitation are available only for 
leading or next to leading PN orders and hence more work is required before they are comparable in performance to the GR waveforms used presently for GW data analysis. Given these circumstances, our purpose is to explore what best we can extract from a subclass of PN models close to GR and leave to future works a more complete investigation using e.g. ppE models.

Based on the above analysis, within the subclass of $\mathrm{ppE}$ theories that we consider, we find that GW observations by advanced LIGO of binary black holes (BBHs) in the range $11-110 M_{\odot}$ and at a luminosity distance of $300 \mathrm{Mpc}$ would allow the measurement of the PN coefficient $\psi_{3}$ with fractional accuracies better that $6 \%$ when the FWF is used. On the other hand using the FWF as a waveform model and a low-frequency cutoff of $1 \mathrm{~Hz}$, observations of stellar mass BBHs in ET would allow the measurement of all $\mathrm{PN}$ parameters, except $\psi_{4}$, with accuracies better than $2 \%$ when the total mass of the binary is in the range $11-44 M_{\odot}$. ET observations of intermediate mass BBHs would allow only two of the seven PN coefficients $\left(\psi_{3}\right.$ and $\psi_{5 l}$ ) to be measured with fractional accuracies better than $10 \%$ in the mass range $55-400 M_{\odot}$.

The choice of a low-frequency cutoff of $1 \mathrm{~Hz}$, as compared to $10 \mathrm{~Hz}$, reduces the relative errors in various parameters roughly by factors of order 2 to 10 for stellar mass black hole binaries. For intermediate mass binaries, which coalesce at smaller frequencies, though a lower cutoff helps improve the parameter estimation, the errors associated with the measurement of various parameters is so large that the test is not very interesting. Although the use of FWF has no particular advantage in the case of stellar mass black hole binaries, their use in the case of intermediate mass black hole binaries improves parameter estimation by a factor of a few to almost 80. Large improvements are obtained for binaries that are more massive than $\sim 100 M_{\odot}$. Error in the estimation of the various PN parameters for such systems is already so great that the improvement brought about by the use of FWF is not useful for the type of tests discussed in this study. For reasonable detection rates, intermediate mass $\mathrm{BH}$ binaries are at distances greater than $3 \mathrm{Gpc}$ as opposed to $300 \mathrm{Mpc}$ in the stellar mass case (see, e.g., Ref. [35]): The gain due to the use of FWF is offset by an order-of-magnitude loss since sources are farther away.

The rest of this paper is organized in the following way: In Sec. II we have introduced the noise curves that are employed for Advanced LIGO and ET and the waveform model used in the present work. This is followed by a brief description of the Fisher matrix formalism that will be used to perform parameter estimation, our proposal for testing GR, the physical systems investigated, and the implementation of the test. In Sec. III, we discuss our results and various issues related to the present work like the systematics due to higher-order PN terms, choice of parametrization used in the test, and the effect of choice of source location on the results. Finally, in Sec. IV we give a summary of our findings and future directions.

\section{TEST OF GR WITH ADVANCED LIGO AND THE EINSTEIN TELESCOPE}

In this work we shall focus on the measurement of various PN coefficients in the context of Advanced LIGO and the Einstein Telescope. One would like to investigate whether the observations of stellar mass black hole binaries in Advanced LIGO (with SNR 30) and observations of stellar mass (with SNR of a few hundreds) as well as intermediate mass (with SNR 40) BBHs in ET, will allow us to measure some of the PN coefficients (if not all) with good accuracies.

The first generation of long baseline interferometric gravitational-wave detectors (GEO600, LIGO, and Virgo) have more or less reached their design sensitivity and have operated for a number of years taking good science quality data. They have shown that it is possible to build, control, and operate highly sensitive instruments. All of these projects are now on the path toward building advanced versions with strain sensitivities of a factor of 10 better than their current versions. This is made possible with research and technology in high power lasers, ultrahigh seismic isolation systems, improved control systems, etc., that has been developed over the past decade. When completed around 2015-2017, advanced detectors are expected to make routine observation of gravitational waves - the most promising of all sources being the coalescence of binaries consisting of compact objects (see Sec. IIE for expected binary coalescence rates).

While advanced detectors will open the gravitational window for astronomical observations, the expected signal-to-noise ratios will not be routinely large enough to carry out strong-field tests of GR or high precision measurements of cosmological measurements. The worldwide gravitational-wave community has already begun to explore the technological development that is necessary to build detectors that are an order of magnitude better than the advanced instruments. The Einstein Telescope is a three-year conceptual design study funded by the European Commission with the goal to identify the challenges to mitigate gravity gradient and seismic noise in the low-frequency region to make it possible to observe in the $1-10 \mathrm{~Hz}$ band. ET will be designed to also make an orderof-magnitude improvement in strain sensitivity in the 10$1000 \mathrm{~Hz}$ band. Such a detector will be capable of making routine observation of high-SNR events that will be useful for carrying out precisions tests of general relativity.

\section{A. Advanced LIGO}

For the studies related to Advanced LIGO we use the Advanced LIGO sensitivity curve [36]. The analytical fit of the noise curve for Advanced LIGO is given by the expression, 


$$
\begin{aligned}
S_{h}(f) & =S_{0}\left[10^{16-4(f-7.9)^{2}}+2.4 \times 10^{-62} x^{-50}+0.08 x^{-4.69}+123.35\left(\frac{1-0.23 x^{2}+0.0764 x^{4}}{1+0.17 x^{2}}\right)\right], \quad f \geq f_{s}, \\
& =\infty, \quad f<f_{s} .
\end{aligned}
$$

where $x=f / f_{0}, f_{0}=215 \mathrm{~Hz}, S_{0}=10^{-49} \mathrm{~Hz}^{-1}$, and $f_{s}$ is a low-frequency cutoff below which $S_{h}(f)$ can be considered infinite for all practical purposes. We have chosen it to be $20 \mathrm{~Hz}$. The amplitude spectrum of Advanced LIGO is plotted in Fig. 1.

\section{B. Einstein Telescope}

The ET design study has come up with a number of possible sensitivity curves [37]. Examples include a single detector that operates with an improved sensitivity over the whole band of $1 \mathrm{~Hz}$ to $1 \mathrm{kHz}$ and a xylophone configuration consisting of a pair of detectors, one tuned for best lowfrequency (i.e., $1-100 \mathrm{~Hz}$ ) sensitivity and a second detector tuned for optimal performance at higher frequencies of $100 \mathrm{~Hz}$ to a few $\mathrm{kHz}$. In our studies in this paper, we will use the ET-B sensitivity curve [37], which is also the official sensitivity curve for ET.

An analytical fit to the ET sensitivity curve is given by

$$
\begin{aligned}
S_{h}^{1 / 2}(f) & =S_{0}^{1 / 2}\left[a_{1} x^{b_{1}}+a_{2} x^{b_{2}}+a_{3} x^{b_{3}}+a_{4} x^{b_{4}}\right], \quad f \geq f_{s}, \\
& =\infty, \quad f<f_{s},
\end{aligned}
$$

where $x=f / f_{0}, f_{0}=100 \mathrm{~Hz}, S_{0}=10^{-50} \mathrm{~Hz}^{-1}$, and $f_{s}$ is a low-frequency cutoff below which $S_{h}(f)$ can be considered infinite for all practical purposes. Also one has

$$
\begin{array}{ll}
a_{1}=2.39 \times 10^{-27}, & b_{1}=-15.64, \\
a_{2}=0.349, & b_{2}=-2.145, \\
a_{3}=1.76, & b_{3}=-0.12, \\
a_{4}=0.409, & b_{4}=1.10 .
\end{array}
$$

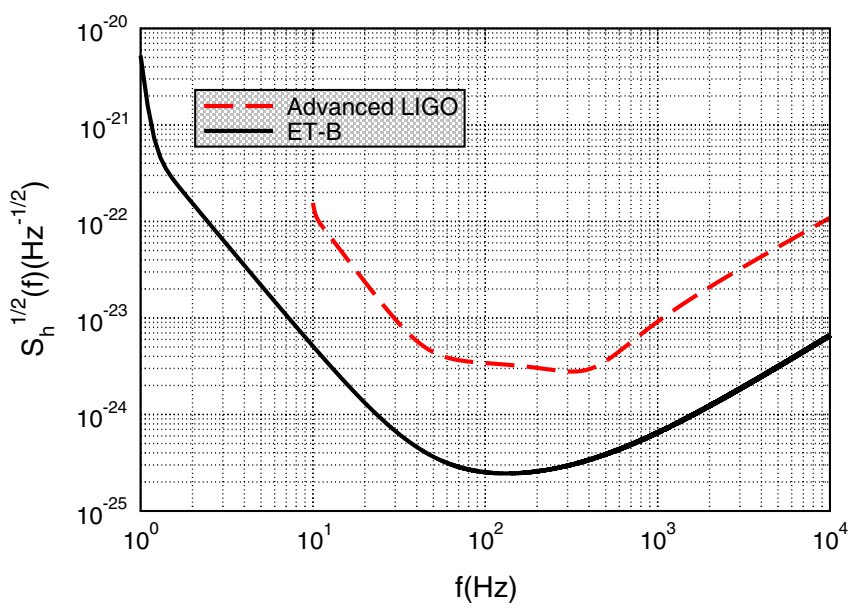

FIG. 1 (color online). Amplitude spectrum of Advanced LIGO and ET.
The amplitude spectrum of ET is plotted in Fig. 1. In connection with the ET design study one of the issues to be looked into is the science case for going down to as low a frequency as $1 \mathrm{~Hz}$ versus a more modest choice of $10 \mathrm{~Hz}$.

\section{The waveform model}

The earlier papers which discussed the tests of GR, including our own papers $[23,25]$, assumed the so-called restricted post-Newtonian waveform (RWF) for quasicircular, adiabatic inspiral, which contains the dominant harmonic at twice the orbital frequency and no corrections to the amplitude. In the present study, we include the effects of subdominant harmonics in the waveforms. Such a waveform, as mentioned earlier, is called the FWF and includes harmonics other than the dominant one, each having PN corrections to their amplitudes. At present the most accurate waveforms available include PN corrections in amplitude to 3PN order and in phase to 3.5PN order [38-40]. To see how one might test GR or, more precisely, the structure of the PN theory, let us begin by considering the waveform from a binary in the frequency domain. The full signal in its general form reads as

$$
\begin{aligned}
\tilde{h}(f)= & \frac{2 M \nu}{D_{L}} \sum_{k=1}^{8} \sum_{n=0}^{6} \frac{A_{(k, n / 2)}\left(t\left(f_{k}\right)\right) x^{(n / 2)+1}\left(t\left(f_{k}\right)\right)}{2 \sqrt{k \dot{F}\left(t\left(f_{k}\right)\right)}} \\
& \times \exp \left[-i \phi_{(k, n / 2)}\left(t\left(f_{k}\right)\right)+2 \pi i f t_{c}-i \pi / 4+i k \Psi\left(f_{k}\right)\right],
\end{aligned}
$$

where $f_{k}=f / k$, and the Fourier phase $\Psi(f)$ is given by

$$
\Psi(f)=-\phi_{c}+\sum_{j=0}^{7}\left[\psi_{j}+\psi_{j l} \ln f\right] f^{(j-5) / 3} .
$$

Here $t_{c}$ and $\phi_{c}$ are the fiducial epoch of merger and the phase of the signal at that epoch, respectively. Quantities appearing in Eq. (2.4) with argument $t\left(f_{k}\right)$ denote their values at the time when the instantaneous orbital frequency $F(t)$ sweeps past the value $f / k$, and $x(t)$ is the PN parameter given by $x(t)=[2 \pi M F(t)]^{2 / 3} \cdot A_{(k, n / 2)}(t)$ and $\phi_{(k, n / 2)}(t)$ are the polarization amplitudes and phases of the $k$ th harmonic at $n / 2$ th PN order in amplitude. The coefficients in the PN expansion of the Fourier phase are given by

$$
\begin{aligned}
& \psi_{j}=\frac{3}{256 \nu}(2 \pi M)^{(j-5) / 3} \alpha_{j}, \\
& \psi_{j l}=\frac{3}{256 \nu}(2 \pi M)^{(j-5) / 3} \alpha_{j l},
\end{aligned}
$$

where 


$$
\begin{aligned}
& \alpha_{0}=1, \quad \alpha_{1}=0, \quad \alpha_{2}=\frac{3715}{756}+\frac{55}{9} \nu, \quad \alpha_{3}=-16 \pi, \quad \alpha_{4}=\frac{15293365}{508032}+\frac{27145}{504} \nu+\frac{3085}{72} \nu^{2} \\
& \alpha_{j l}=
\end{aligned}
$$

The constant $C=0.577 \cdots$, appearing in the expression for $\alpha_{6}$, is Euler's constant.

We have total nine post-Newtonian parameters; seven of these are the coefficients of $v^{n}$ terms for $n=0,2,3,4$, 5, 6, 7 and two are coefficients of $v^{n} \ln v$ terms for $n=5,6$. These are PN coefficients in Einstein's theory and are functions of just two mass parameters chosen to be the total mass $M$ and symmetric mass ratio $\nu$.

In addition to mass dependence, the amplitude corrections also depend on the luminosity distance of the source to the observer and four additional angular parameters $(\cos \theta, \phi, \psi, \cos \iota)$ related to the source location and orientation [41,42]: $\theta$ and $\phi$ determine the sources location, $\psi$ is the polarization angle, and $\iota$ is the inclination angle. Once the mass dependences in amplitude corrections are replaced by our fundamental pair of $\psi_{0}$ and $\psi_{2}$, the whole waveform can be characterized by a total ten parameters

$$
\mathbf{p} \equiv\left(\ln D_{L}, t_{c}, \phi_{c}, \psi_{0}, \psi_{2}, \psi_{T}, \cos \theta, \phi, \psi, \cos \iota\right)
$$

\section{Fisher matrix and statistical errors}

We employ the Fisher matrix approach $[43,44]$ to see how well we can measure these parameters. Below we briefly list the basic equations of the Fisher matrix approach that we subsequently need.

Let $\tilde{\theta}^{a}$ denote the "true values" of the parameters and let $\tilde{\theta}^{a}+\Delta \theta^{a}$ be the best-fit parameters in the presence of some realization of the noise. Then for large SNR, error in the estimation of parameters $\Delta \theta^{a}$ obeys a Gaussian probability distribution [43-46] of the form

$$
p\left(\Delta \theta^{a}\right)=p^{(0)} \exp \left[-\frac{1}{2} \Gamma_{b c} \Delta \theta^{b} \Delta \theta^{c}\right],
$$

where $p^{(0)}$ is a normalization constant. The quantity $\Gamma_{a b}$ appearing in Eq. (2.9) is the Fisher information matrix and is given by

$$
\Gamma_{a b}=\left(h_{a} \mid h_{b}\right),
$$

where $h_{a} \equiv \partial h / \partial \theta^{a}$. Here, ( I ) denotes the noise weighted inner product. Given any two functions $g$ and $h$ their inner product is defined as

$$
(g \mid h) \equiv 4 \operatorname{Re} \int_{f_{\min }}^{f_{\max }} d f \frac{\tilde{g}^{*}(f) \tilde{h}(f)}{S_{h}(f)} .
$$

The integration limit $\left[f_{\min }, f_{\max }\right]$ is determined by both the detector and by the nature of the signal. Each harmonic in $\tilde{h}(f)$ is assumed to vanish outside a certain frequency range. The simplest physical choice is to set the contribution from the $k$ th harmonic to the waveform zero above the frequency $k f_{\text {lso }}$, where $f_{\text {lso }}$ is the orbital frequency at the last stable orbit [47]. Since the amplitude-corrected waveform we are using in this work has eight harmonics, we set the upper cutoff to be $8 f_{\text {lso }}$ when we use the FWF in the analysis. For lower cutoff, as power spectral densities $S_{h}(f)$ tend to rise very quickly below a certain frequency $f_{s}$ where they can be considered infinite for all practical purposes, we may set it to be $f_{s}$. Using the definition of the inner product one can reexpress $\Gamma_{a b}$ more explicitly as

$$
\Gamma_{a b}=4 \int_{f_{s}}^{k f_{\text {lso }}} \frac{\operatorname{Re}\left(\tilde{h}_{a}^{*}(f) \tilde{h}_{b}(f)\right)}{S_{h}(f)} d f .
$$

The covariance matrix, defined as the inverse of the Fisher matrix, is given by

$$
\Sigma^{a b} \equiv\left\langle\Delta \theta^{a} \Delta \theta^{b}\right\rangle=\left(\Gamma^{-1}\right)^{a b},
$$

where $\langle\cdot\rangle$ denotes an average over the probability distribution function in Eq. (2.9). The root-mean-square error $\sigma_{a}$ in the estimation of the parameters $\theta^{a}$ is

$$
\sigma_{a}=\left\langle\left(\Delta \theta^{a}\right)^{2}\right\rangle^{1 / 2}=\sqrt{\sum^{a a}} .
$$

In the present work we deal with inspiralling compact binaries as seen by Earth bound detectors. For such burst sources, one can approximate the detector's beam pattern functions as being constant over the duration of the signal and thus we can assume that angular parameters $(\cos \theta, \phi$, and $\psi)$ as well as the luminosity distance $\left(D_{L}\right)$ are fixed and thus can be excluded from the analysis. With this restriction, the large 10-dimensional parameter space reduces to a smaller 6-dimensional parameter space given by

$$
\mathbf{p} \equiv\left(t_{c}, \phi_{c}, \psi_{0}, \psi_{2}, \psi_{T}, \cos \iota\right)
$$

In order to test the PN structure, one should be able to measure various PN coefficients with good accuracy. In the 
present work we have assumed that the relative error in the measurement of a parameter should be less than $10 \%$, i.e. $\Delta \psi_{j} / \psi_{j} \leq 0.1$, where $\Delta \psi_{j}$ is the error in the estimation of the parameter $\psi_{j}$, in order to estimate its value in the PN series with confidence.

\section{E. Systems investigated}

The first detection of gravitational radiation in groundbased interferometric detectors is generally expected to be from the coalescence of compact binary systems with neutron star and black hole components [48]. Among these, binary neutron stars (BNS) are arguably the most promising ones with expected rates of about 40 mergers per year in Advanced LIGO and millions of them in ET. While very interesting for other proposed tests of GR, BNS systems are not useful for the tests proposed in this study. For our purposes a compact binary in which one or both the components is a stellar mass $\left(\sim 2-30 M_{\odot}\right)$ or intermediate mass $\left(\sim 50-1000 M_{\odot}\right)$ black hole (the other being a neutron star) would be most interesting. For our studies related to Advanced LIGO, we have chosen binary black holes in the mass range $11-110 M_{\odot}$ and their distance from the Earth to be $300 \mathrm{Mpc}$.

For the analysis using ET we have discussed separately stellar mass and intermediate mass BBHs. For stellar mass BBHs, we have again chosen their luminosity distance from the Earth to be $300 \mathrm{Mpc}$ and the range of the total mass to be $11-44 M_{\odot}$. Coalescence rate of stellar mass BBHs is highly uncertain. The predicted rate of coalescence within a distance of $300 \mathrm{Mpc}$ varies between one event per 10 years to several per year [49]. However, it is with such rare high-SNR events that one expects to perform precision tests of GR. For intermediate mass black holes, we have chosen the distance to be $3 \mathrm{Gpc}(z=0.55)$, and their total mass to be in the range $55-1100 M_{\odot}$. The evolutionary history of intermediate mass BBHs and their rate of coalescence is still not well understood. The main motivation to study these systems comes from the models that invoke them as seeds of massive black holes at galactic nuclei. In a recent study, it has been suggested that only few coalescence events of intermediate mass BBHs could be expected within a redshift of $z=2$. Also depending on what triggered seed galaxies there may be a few events within a redshift of $z=1[35,49-51]$.

\section{F. Implementation of the test}

As mentioned earlier, in Einstein's theory (and thus in theories "close" to GR) each PN coefficient for a nonspinning compact binary is a function of the two mass parameters, the total mass $M$ and the symmetric mass ratio $\nu$. In other words, we can say that each $\psi_{i}$ is a function of the masses $\left(m_{1}, m_{2}\right)$ of the components constituting the binary, i.e. $\psi_{i} \equiv \psi_{i}\left(m_{1}, m_{2}\right)$. With high-SNR GW observations of stellar and intermediate mass $\mathrm{BBHs}$ in Advanced LIGO and ET, it would be possible to measure the individual masses constituting the binary with good accuracies. Thus, once the (statistical) error in the parameter is estimated using the Fisher matrix, we can represent the region it spans in the space of masses by inverting the relation $\psi_{i} \equiv \psi_{i}\left(m_{1}, m_{2}\right)$ to get say $m_{2} \equiv m_{2}\left(\psi_{i}, m_{1}\right)$. Given the measured value $\psi_{i}^{\text {meas }}$ and the errors $\Delta \psi_{i}$ in the estimation of $\psi_{i}$, the region in the mass plane corresponding to $m_{2}$ is given by $m_{2} \equiv m_{2}\left(\psi_{i}^{\text {meas }} \pm \Delta \psi_{i}, m_{1}\right)$. For each $\psi_{i}$, there would be an allowed region in the $m_{1}-m_{2}$ plane and if Einstein's theory of gravity, or, more precisely, the PN approximation to it, is a correct theory then the three parameters $\psi_{0}, \psi_{2}$, and $\psi_{T}$ (the test parameter) should have a common nonempty intersection in the $m_{1}-m_{2}$ plane. Proceeding in this way, for six test parameters we shall have six different tests of the theory. In the present work, we shall only discuss asymmetric binaries with component mass ratio $q_{m}=0.1$. Since the different PN coefficients are symmetric with respect to the exchange of $m_{1}$ and $m_{2}$, we expect plots in the $m_{1}-m_{2}$ plane to have two symmetric branches. Figures 2, 6, and 8 show one branch of the full plot.

Figure 2 schematically demonstrates how the test works by using $\psi_{0}$ and $\psi_{2}$ as basic variables and $\psi_{5 l}$ as a test parameter. The plot on the left uses PN coefficients predicted by GR, assuming GR is a correct theory of gravity. Clearly, this shows that all three parameters, $\psi_{0}, \psi_{2}$, and $\psi_{5 l}$, have a common nonempty intersection in the plane of masses and this is what we expect if GR is the correct theory of gravity.

In contrast, consider the possibility that the correct theory of gravity is a hypothetical non-GR theory in which the phasing coefficient $\psi_{5 l}$ and all higher PN coefficients differ from the GR values by $1 \%$. We have assumed here, in

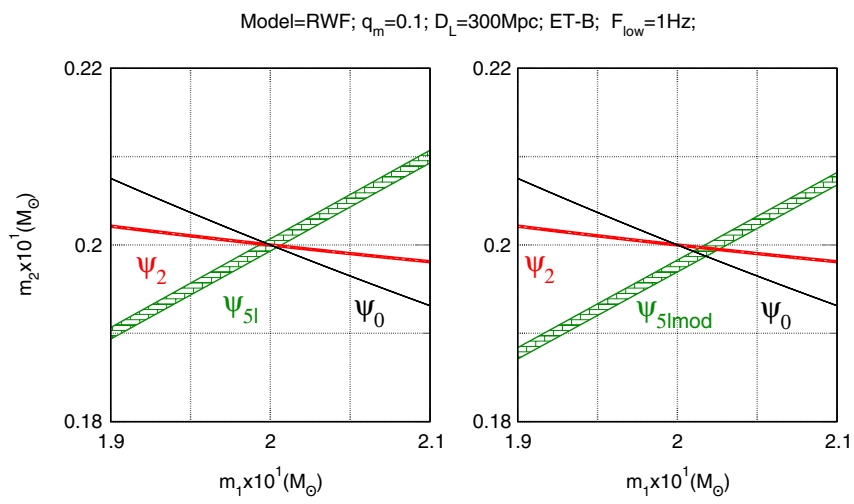

FIG. 2 (color online). Plots showing the regions in the $m_{1}-m_{2}$ plane that correspond to 1- $\sigma$ uncertainties in $\psi_{0}, \psi_{2}$, and $\psi_{5 l}$ (left panel) and those in $\psi_{0}, \psi_{2}$, and $\psi_{5 \text { lmod }}$ (right panel) for a $(2,20) M_{\odot} \mathrm{BBH}$ at a luminosity distance of $D_{L}=300 \mathrm{Mpc}$ observed by ET. The low-frequency cutoff is $1 \mathrm{~Hz}$ and RWF has been used. For the curves in the right panel we have assumed that the correct theory of gravity is a hypothetical non-GR theory in which the phasing coefficient $\psi_{5 l}$ and all higher PN coefficients differ from the GR values by $1 \%$. 
Model=RWF; $\mathrm{q}_{\mathrm{m}}=0.1 ; \mathrm{D}_{\mathrm{L}}=3 \mathrm{Gpc} ; \mathrm{ET}-\mathrm{B} ; \mathrm{F}_{\text {low }}=1 \mathrm{~Hz}$;
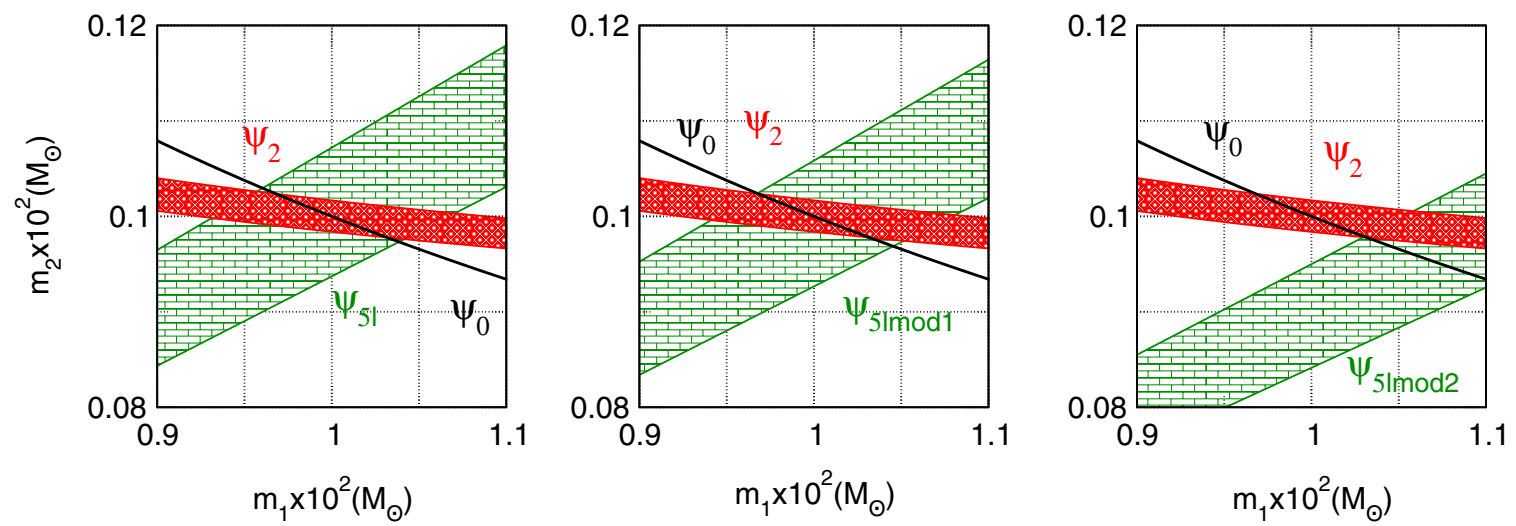

FIG. 3 (color online). Plots showing the regions in the $m_{1}-m_{2}$ plane that correspond to 1- $\sigma$ uncertainties in Newtonian, 1PN, and $2.5 \mathrm{PN}$ coefficients in the PN series for a $(10,100) M_{\odot} \mathrm{BBH}$ at a luminosity distance of $D_{L}=3$ Gpc observed by ET. The lowfrequency cutoff is $1 \mathrm{~Hz}$ and RWF has been used. The left panel corresponds to GR as the correct theory of gravity while the middle and right panels correspond to hypothetical non-GR theories of gravity which have phasing coefficients $\psi_{5 l}(2.5 \mathrm{PN})$ and higher differing from the GR values by $1 \%$ and $10 \%$, respectively.

an ad hoc manner, that the deviation of the PN terms at higher orders above the $2.5 \mathrm{PN}$ term (which is put to test) to be a simple scaling, i.e., $\psi_{k} \rightarrow 1.01 \psi_{k}$ for $k \geq 5$. As we shall show later, there is a range of binary masses for which the observation of the GW signal by ET could estimate this coefficient with an accuracy much better than $1 \%$. In this scenario, if we interpret the $\psi_{5 l}$ obtained by fitting to the observed GW signal, as a GR coefficient, there will definitely be an inconsistency in the $m_{1}-m_{2}$ plane. This can clearly be seen in the right panel of Fig. 2 where there is no overlapping region between the three parameters in question in the $m_{1}-m_{2}$ plane, thus demonstrating the spirit of the proposed test.

Figure 3 shows a similar exercise for the $(10,100) M_{\odot}$ $\mathrm{BBH}$ located at a luminosity distance of $D_{L}=3 \mathrm{Gpc}$ observed by ET. The low-frequency cutoff is $1 \mathrm{~Hz}$ and RWF has been used. The left panel in Fig. 3 assumes that GR is the correct theory of gravity, whereas the middle and right panels assume that the correct theory of gravity is a hypothetical non-GR theory in which the PN coefficients at 2.5PN (i.e., $\psi_{5 l}$ ) and all higher orders differ from their GR values by $1 \%$ and $10 \%$, respectively. The $2.5 \mathrm{PN}$ coefficient in GR and in the above two hypothetical theories can be measured with fractional accuracies of $5.6 \%, 5.5 \%$, and $5.1 \%$, respectively, for the system under consideration.

As in the previous case (cf., Fig. 2), we notice the departures from GR. However, these departures are not very clear in the middle panel where the $2.5 \mathrm{PN}$ coefficient and other higher-order terms differ from their GR values by only $1 \%$. As a result one would not be able to discriminate between the two theories by using GW observations of such sources in ET, even though the parameter can be measured accurately. The right panel of Fig. 3, which corresponds to a theory in which the values of $2.5 \mathrm{PN}$ and higher-order coefficients differ from their GR values by
$10 \%$, brings such significant departures from GR that despite the larger errors in the estimation of the test parameter, one can distinguish between the two theories using the test we are proposing here. One should bear in mind that the model we have used is the simplest possible way in which a deviation could occur. But our purpose here is to have a proof of principle demonstration of the power of the proposed test, given the lack of knowledge of the exact manner in which such deviations could occur.

To summarize, assuming that GR is correct, our proposal gives the accuracy with which three of the PN parameters can be measured. How does that test GR? For e.g. if GR is not correct and differs at, say 1.5 PN level onward, then our claim is that we would begin to see inconsistencies in the estimated parameter values beyond the accuracy of measurement provided deviations from GR are large enough. One may be concerned about the extent to which the departure of higher-order terms from their GR values would penalize the estimation of lower-order terms. As evidenced by our examples above, they induce bias in the estimation of parameters but do not lead to greater errors in the estimation of parameters. In other words, the 1.5PN and higher-order PN coefficients not agreeing with GR might shift the mean of the distribution of $(M, \nu)$ but the width should remain more or less the same. Put differently, if the PN expansion differs from GR slightly then the error in the estimation of parameters will not change to first order.

\section{THE RESULTS}

\section{A. Advanced LIGO}

In this section we investigate the possibility of the test using GW observations of BBHs in Advanced LIGO. As discussed earlier, the range of total mass explored is 

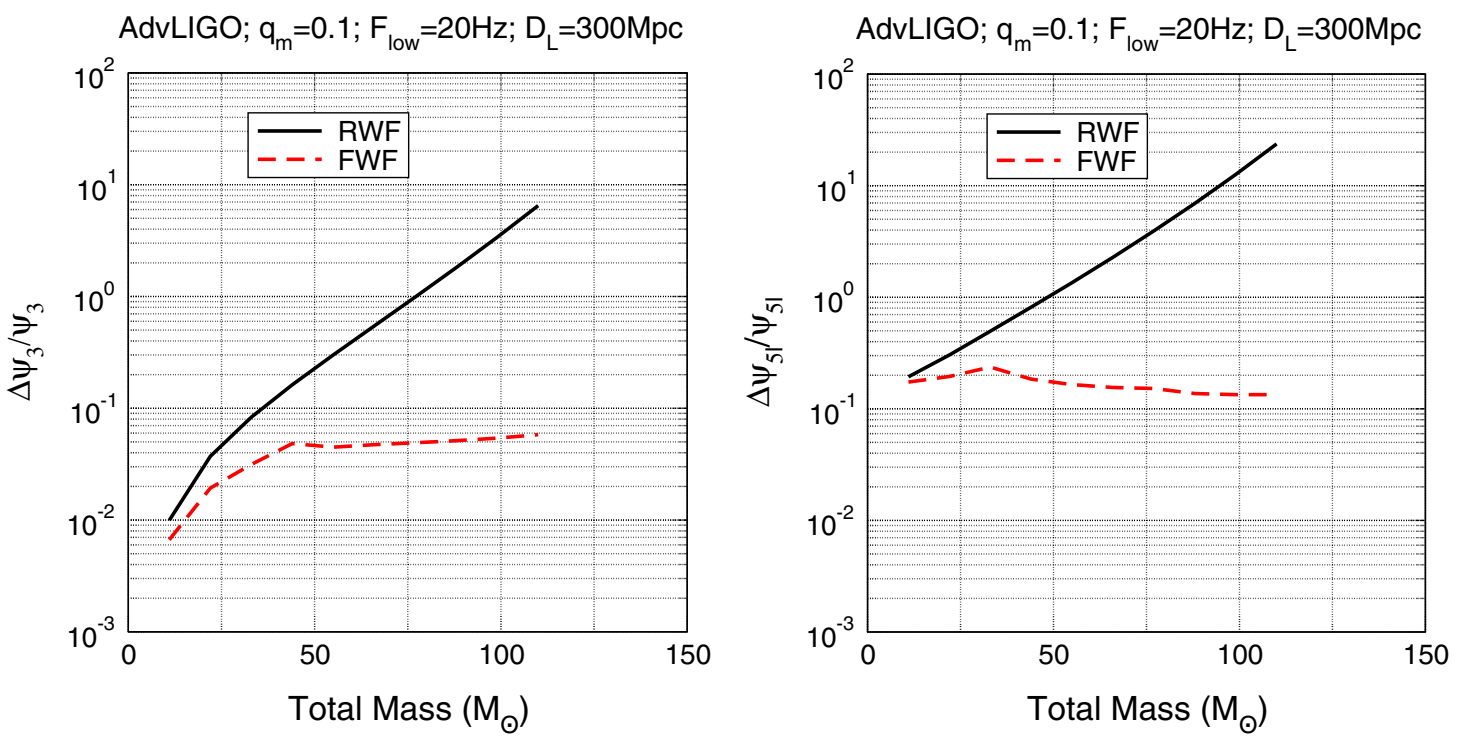

FIG. 4 (color online). Plots showing the variation of relative errors $\Delta \psi_{T} / \psi_{T}$ in the test parameters $\psi_{T}=\psi_{3}, \psi_{5 l}$ as a function of total mass of binaries in the range $11-110 M_{\odot}$ (with component masses having mass ratio of 0.1 ) located at $300 \mathrm{Mpc}$ observed by Advanced LIGO, using both the RWF and the FWF as a waveform model with the source orientations chosen arbitrarily to be $\theta=$ $\phi=\pi / 6, \psi=\pi / 4$, and $\iota=\pi / 3$. The noise curve corresponds to the one shown in Fig. 1 for the Advanced LIGO case and its analytical fit is given by Eq. (2.1). It is evident from the plot in the left panel that the fractional accuracies with which $\psi_{3}$ can be measured are better than $6 \%$ for the entire mass range under consideration when FWF is used and thus can be used to test the theory of gravity. $\psi_{5 l}$ (right panel) can be measured with fractional accuracies better than $23 \%$ for the entire mass range when FWF is used but being a poorly determined parameter it can provide a much less stringent test of the theory of gravity.

$11-110 M_{\odot}$ and we assume that binaries are located at a luminosity distance of $300 \mathrm{Mpc}$. Plots in Fig. 4 show the variation of relative accuracies with which two of the PN coefficients, $\psi_{3}$ and $\psi_{5 l}$, can be measured by Advanced LIGO using the restricted and the full waveform. The components of the binary have the mass ratio of 0.1 .

It is evident from the plots that when the FWF is used, $\psi_{3}$ and $\psi_{5 l}$ can be measured with fractional accuracies better than $6 \%$ and $23 \%$, respectively, in the whole mass range under consideration. We shall require (rather arbitrarily) that the relative error in the measurement of a PN coefficient be less than $10 \%$ in order for the test to be effective. Clearly, $\psi_{3}$ can be estimated quite accurately and thus it can be used to test the theory. On the other hand, since $\psi_{5 l}$ is not so well determined, it can still provide a less stringent test of the theory. The measurement of other PN coefficients is not accurate enough to lead to a meaningful test of GR.

The plots clearly show the benefits of bringing higher harmonics into the analysis. The use of the FWF typically improves the estimation by a factor of 3 to almost 100 .

\section{B. Einstein Telescope}

In the previous section we have seen that with Advanced LIGO one can only test PN theory up to 1.5PN. Can one do better with the proposed third generation detector like the ET? In what follows we investigate the extent to which one can test the PN theory using GW observations of stellar mass and intermediate mass BBHs using ET. In addition to this we will discuss some other key issues influencing the results such as effects of PN systematics on the test, choice of parametrization, and dependence of the test on angular parameters.

\section{Stellar mass black hole binaries}

Figure 5 plots the relative errors $\Delta \psi_{T} / \psi_{T}$ as a function of total mass $M$ of the binary at a distance of $D_{L}=$ $300 \mathrm{Mpc}$. We have considered stellar mass BBHs of unequal masses and mass ratio 0.1 , with the total mass in the range $11-44 M_{\odot}$. Figure 5 also shows two types of comparisons: (a) full waveform vs restricted waveform, and (b) a lower frequency cutoff of $10 \mathrm{vs} 1 \mathrm{~Hz}$. The top and bottom panels correspond to the lower frequency cutoff of 1 and $10 \mathrm{~Hz}$, respectively, while the left and right panels correspond to the RWF and FWF, respectively. The source orientations are chosen arbitrarily to be $\theta=\phi=\pi / 6$, $\psi=\pi / 4$, and $\iota=\pi / 3$. It should be evident from the plots that the best estimates of various test parameters are for the combination using the FWF with a lower cutoff frequency of $1 \mathrm{~Hz}$. In this case, all $\psi_{i}$ 's except $\psi_{4}$ can be measured with fractional accuracies better that $2 \%$ for the total mass in the range $11-44 M_{\odot}$. On the other hand when the lower cutoff is $10 \mathrm{~Hz}$, with the FWF all $\psi_{i}$ 's except $\psi_{4}$ can be measured with fractional accuracies better than $7 \%$. It is also evident from the plots that as compared to other test parameters, $\psi_{3}$ is the most accurately measured parameter in all cases and best estimated when the lower frequency cutoff is $1 \mathrm{~Hz}$. On the other hand, $\psi_{4}$ is the worst 



FIG. 5 (color online). Plots showing the variation of relative errors $\Delta \psi_{T} / \psi_{T}$ in the test parameters $\psi_{T}=\psi_{3}, \psi_{4}, \psi_{5 l}, \psi_{6}, \psi_{6 l}$, and $\psi_{7}$ as a function of total mass $M$ for stellar mass black hole binaries (with component masses having mass ratio 0.1 ) at a luminosity distance of $D_{L}=300 \mathrm{Mpc}$ observed by ET, using both RWF (left panels) and FWF (right panels) as waveform models. The choice of the source orientations is the same as quoted in Fig. 4. The noise curve corresponds to the recent ET-B sensitivity curve. Top panels correspond to the lower frequency cutoff of $1 \mathrm{~Hz}$. By using FWF as the waveform model all $\psi_{k}$ 's except $\psi_{4}$ can be tested with fractional accuracy better than $2 \%$ in the mass range $11-44 M_{\odot}$. Bottom panels correspond to the lower frequency cutoff of $10 \mathrm{~Hz}$. Using FWF, all $\psi_{k}$ 's except $\psi_{4}$ can be tested with fractional accuracy better than $7 \%$ in the mass range $11-44 M_{\odot}$.

measured parameter of all the test parameters. However, we see the best improvement in its measurement when going from the RWF to the FWF.

Figure 6 shows the regions in the $m_{1}-m_{2}$ plane that corresponds to $1-\sigma$ uncertainties in $\psi_{0}, \psi_{2}$ and various test parameters which in turn will be one of the six test parameters $\psi_{T}=\psi_{3}, \psi_{4}, \psi_{5 l}, \psi_{6}, \psi_{6 l}$, and $\psi_{7}$, one at a time, for a $(2,20) M_{\odot} \mathrm{BBH}$, at a luminosity distance of $D_{L}=300 \mathrm{Mpc}$ observed by ET. It is evident from the plots corresponding to various tests that each test parameter is consistent with corresponding fundamental pair $\left(\psi_{0}, \psi_{2}\right)$.

\section{Intermediate mass black hole binaries}

Figure 7 plots the relative errors $\Delta \psi_{T} / \psi_{T}$ as a func tion of the total mass $M$ of the binary at a distance of $D_{L}=3 \mathrm{Gpc}$. We have considered $\mathrm{BBH}$ of unequal masses with mass ratio 0.1. As in Fig. 5, Fig. 7 also shows two types of comparisons: (a) the effect of the use of FWF on parameter estimation against RWF, and (b) the effect of 

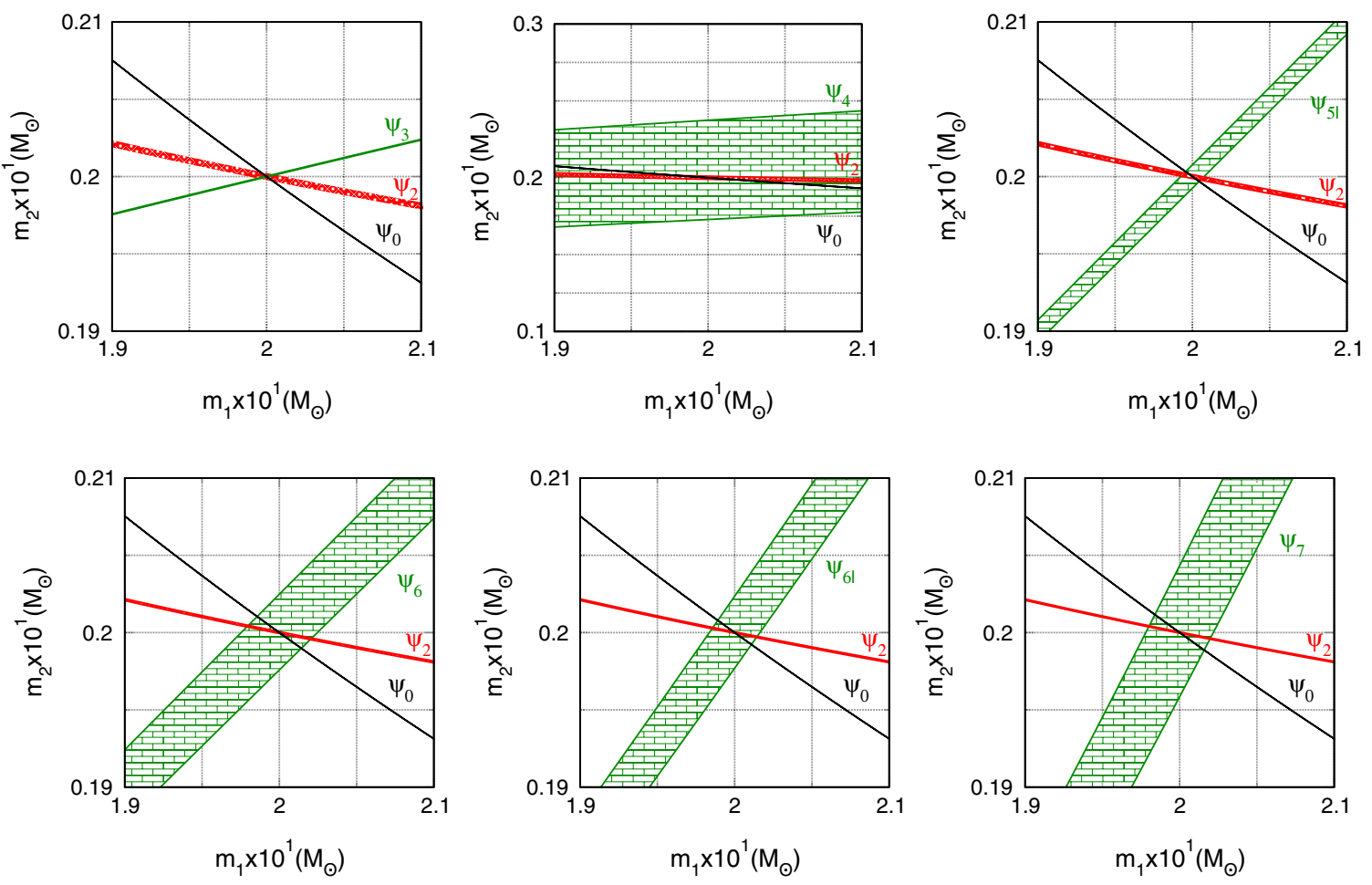

FIG. 6 (color online). Plots showing the regions in the $m_{1}-m_{2}$ plane that correspond to 1- $\sigma$ uncertainties in $\psi_{0}, \psi_{2}$ and various test parameters, which happen to be one of the six test parameters $\psi_{T}=\psi_{3}, \psi_{4}, \psi_{5 l}, \psi_{6}, \psi_{6 l}$, and $\psi_{7}$ at one time, for a (2, 20) $M_{\odot} \mathrm{BBH}$ at a luminosity distance of $D_{L}=300 \mathrm{Mpc}$ observed by ET. In all six plots shown above $\psi_{0}$ and $\psi_{2}$ are chosen as the fundamental parameters (from which we can measure the masses of the two black holes). Each parameter corresponds to a given region in the $m_{1}-m_{2}$ plane and if GR is the correct theory of gravity then all three parameters, $\psi_{0}, \psi_{2}$, and $\psi_{T}$ should have a nonempty intersection in the $m_{1}-m_{2}$ plane. A smaller region leads to a stronger test. Notice that all panels have the same scaling except the top middle panel in which the $Y$ axis has been scaled by a factor 10 .

lowering the cutoff frequency from 10 to $1 \mathrm{~Hz}$. As before, the top and bottom panels correspond to the cutoff frequency of 1 and $10 \mathrm{~Hz}$, respectively, and left and right panels to RWF and FWF, respectively. The source orientations are chosen arbitrarily to be $\theta=\phi=\pi / 6, \psi=\pi / 4$, and $\iota=\pi / 3$.

It is evident from the plots that the least relative errors in various test parameters are for the combination that uses the FWF and a lower cutoff of $1 \mathrm{~Hz}$. Unlike the case of stellar mass BBHs, in the case of intermediate mass BBHs only two of the test parameters, $\psi_{3}$ and $\psi_{5 l}$, can be measured with fractional accuracies better that $10 \%$ for the total mass in the range 55-400M $M_{\odot}$ with FWF and lower cutoff frequency as $1 \mathrm{~Hz}$. On the other hand, when the lower frequency cutoff is $10 \mathrm{~Hz}$ the use of the FWF allows the estimation of $\psi_{3}$ and $\psi_{5 l}$ with fractional accuracies better than $10 \%$ for the total mass in the range $90-220 M_{\odot}$. As compared to other test parameters, $\psi_{3}$ is the most accurately measured parameter in all cases and best estimated when the low-frequency cutoff is $1 \mathrm{~Hz}$. Parameters $\psi_{4}$ and $\psi_{6}$ are poorly measured as compared to the other test parameters but again we see the best improvement in the estimate of $\psi_{4}$ when using the FWF.

Figure 8 shows the regions in the $m_{1}-m_{2}$ plane that correspond to 1- $\sigma$ uncertainties in $\psi_{0}, \psi_{2}$ and the test parameters $\psi_{T}=\psi_{3}, \psi_{4}, \psi_{5 l}, \psi_{6}, \psi_{6 l}$, and $\psi_{7}$, one at a time, for a $(20,200) M_{\odot}$ BBH at a luminosity distance of $D_{L}=3 \mathrm{Gpc}$ observed by ET. It is clear from the plots that each test parameter is consistent with the corresponding fundamental pair $\left(\psi_{0}, \psi_{2}\right)$.

\section{Effects of PN systematics on the test}

The inability to measure all the PN parameters simultaneously led us to propose a more modest procedure to test the PN parameters one at a time. In parameter estimation, it seems intuitive not to ignore our knowledge of the known high PN order phasing. Further, it is natural to assume that if an alternative theory of gravitation, similar to GR, agrees with GR at some PN order, it would agree with it at a lower PN order but may differ from it at some higher PN order. Thus, when testing a coefficient at some particular PN 

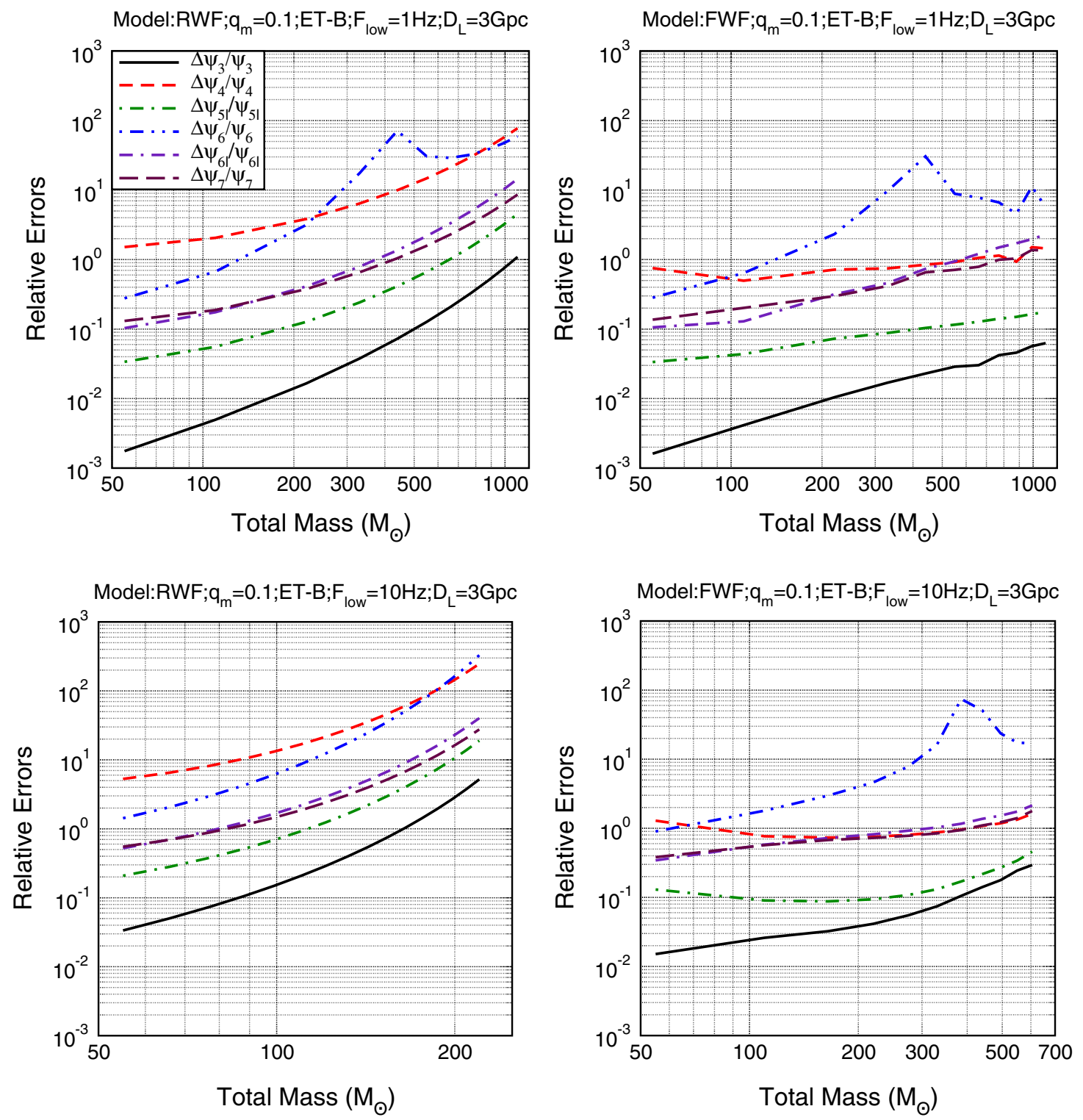

FIG. 7 (color online). Same as Fig. 5 but for intermediate mass black hole binaries (with component masses having mass ratio 0.1) at a luminosity distance of $D_{L}=3 \mathrm{Gpc}$. With lower frequency cutoff of $1 \mathrm{~Hz}$, using FWF as the waveform model, $\psi_{3}$ and $\psi_{5 l}$ can be tested with fractional accuracy better than $10 \%$ for the mass range $55-400 M_{\odot}$. On the other hand, with a lower frequency cutoff of $10 \mathrm{~Hz}$, using the FWF, $\psi_{3}$ and $\psi_{5 l}$ can be tested with fractional accuracy better than $10 \%$ for the mass range $90-220 M_{\odot}$.

order, expressing a lower-order PN coefficient in terms of the basic pair of PN variables seems reasonable. However, expressing the higher PN order coefficients in terms of the basic pair may appear more disconcerting. Here we look at the issue in a little more detail and provide our point of view.

We propose a comparison of the following two schemes: The first scheme, as before, uses $\psi_{0}$ and $\psi_{2}$ as basic parameters and all known PN coefficients up to 3.5PN, except the test parameter, are expressed in terms of $\psi_{0}$ and $\psi_{2}$. The second scheme is similar but the phase evolution is truncated at the PN order corresponding to the test parameter.

Thus, to test $\psi_{3}$ in the second scheme the phasing is truncated at $1.5 \mathrm{PN}$, to test $\psi_{4}$ at $2 \mathrm{PN}$ and so on. Figure 9 compares the two schemes. It should be evident from the figure that in the first scheme the use of 3.5PN phasing, rather than a lower PN order (e.g., 1.5PN in testing $\psi_{3}$ ), does improve the accuracy with which one can measure a certain parameter. Conversely, the poorer estimate (i.e. larger error) in the second scheme is due to the neglect of higher PN order terms. 

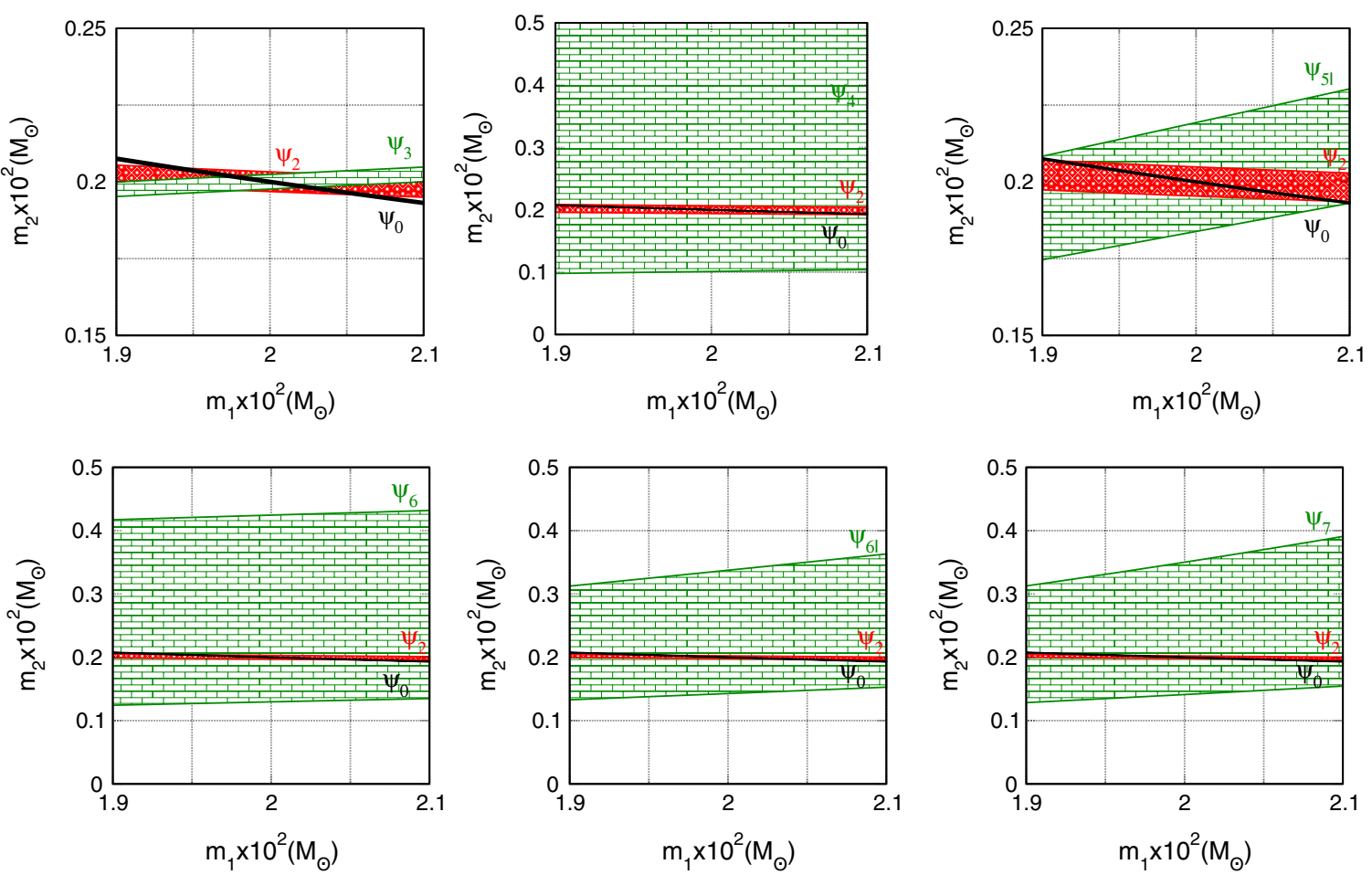

FIG. 8 (color online). Same as in Fig. 6 but for intermediate black hole binaries in the mass range $(20,200) M_{\odot}$ at a luminosity distance of $D_{L}=3 \mathrm{Gpc}$ observed by ET. Notice that all bottom panels and the top middle panel have the same scaling, whereas the $Y$ axes of the top left panel and the top right panel have been scaled by a factor of 5 . Note that there appears just one boundary for $\psi_{4}$ in the plot shown in the top middle panel since the other bound does not exist for the range of values on the $X$ axis.

One can infer, therefore, that what is achieved in the first scheme is an improvement in parameter estimation arising from higher-order PN phasing; parametrization of higherorder PN coefficients in terms the two basic variables is indeed a reasonable choice if one wants to quantitatively look at the deviation from standard GR of an alternative theory of gravitation similar to GR. ${ }^{2}$ Let us also note that if a theory of gravity deviates from GR at a particular PN order, then in our first scheme the test may actually fail at a lower PN order. As a result, our test will not be able to conclusively assert the PN order that is inconsistent with GR. Rather a failure of our test is indicative of the failure of GR at some PN order. It would then be necessary to carry out a more powerful test of the theory by treating all the PN coefficients as independent parameters. Such a test could in principle help determine at which order(s) the true theory of gravity is inconsistent with GR.

\footnotetext{
${ }^{2} \mathrm{~A}$ variant of the test starting from GW phasing expressions in a more general meta theory may need to be implemented to include theories with different PN structure like scalar-tensor theories with qualitatively different effects like dipole radiation.
}

\section{The choice of basic parametrization and the accuracy of the test}

As discussed in Sec. I, in the present work we have chosen the lowest-order (and hence the best determined) PN coefficients $\psi_{0}$ and $\psi_{2}$ to parametrize the waveform. One might wonder whether the choice of $\psi_{0}$ and $\psi_{2}$ as basic variables is the most optimal. To investigate this further, we explored other choices of the basic pair to parametrize the waveform, e.g. $\left(\psi_{0}, \psi_{f}\right)$, where $f$ can be one of $(3,4,5 l, 6,6 l, 7)$. Table I shows a comparison of the accuracies of the measurement of the various PN parameters under different choices of the parametrization schemes for a $(10,100) M_{\odot}$ binary, located at a luminosity distance of $3 \mathrm{Gpc}$. The comparison uses the RWF, with lower frequency cutoffs of 1 and $10 \mathrm{~Hz}$, respectively.

From the table the following observations are evident:

(1) A comparison of the values in blocks symmetric across the principal diagonal one can compare the errors in the estimation of a particular parameter in the following two cases: once when the parameter is one of the basic variables and secondly when it is a test variable. It is also clear that, in general, a parameter is determined more precisely when it is 
Model:RWF; $\mathrm{q}_{\mathrm{m}}=0.1 ; \mathrm{ET}-\mathrm{B} ; \mathrm{F}_{\text {low }}=10 \mathrm{~Hz} ; \mathrm{D}_{\mathrm{L}}=3 \mathrm{Gpc}$
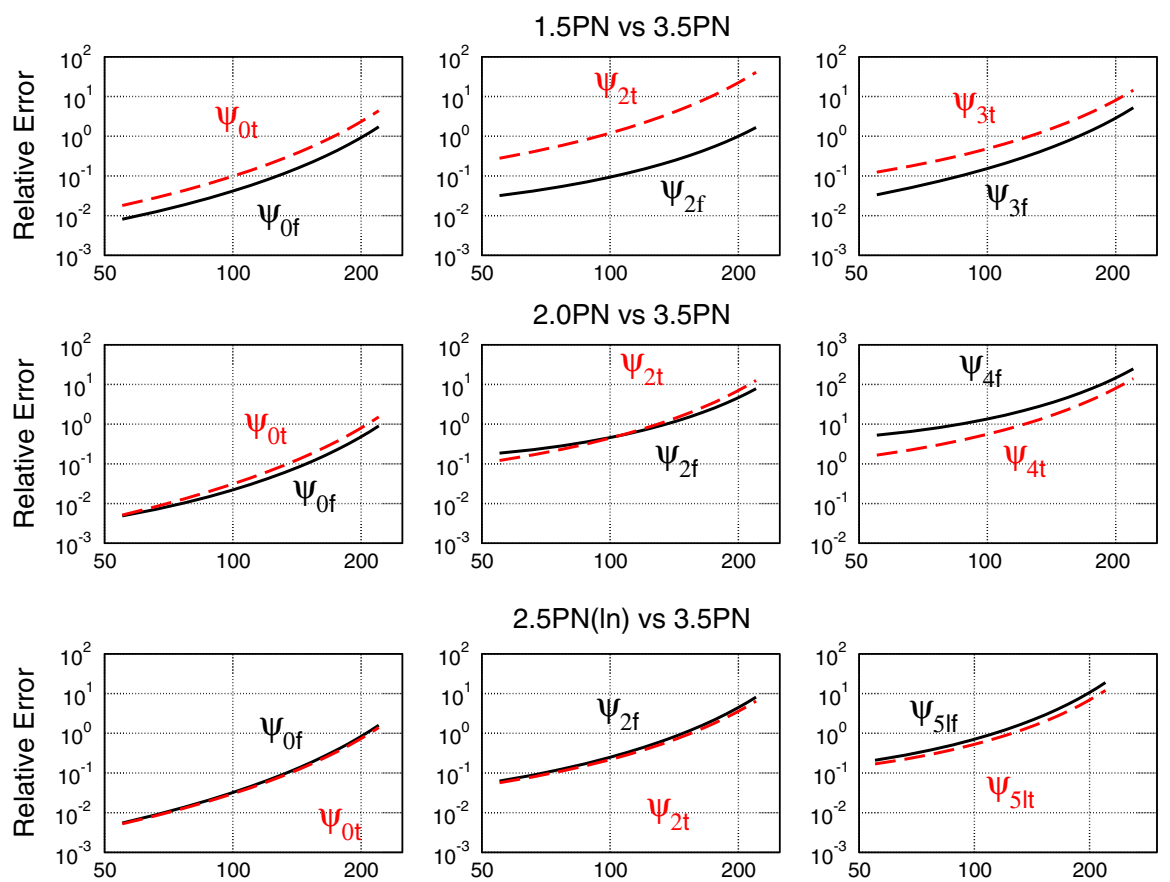

$2.5 \mathrm{PN}(\ln )$ vs 3.5PN
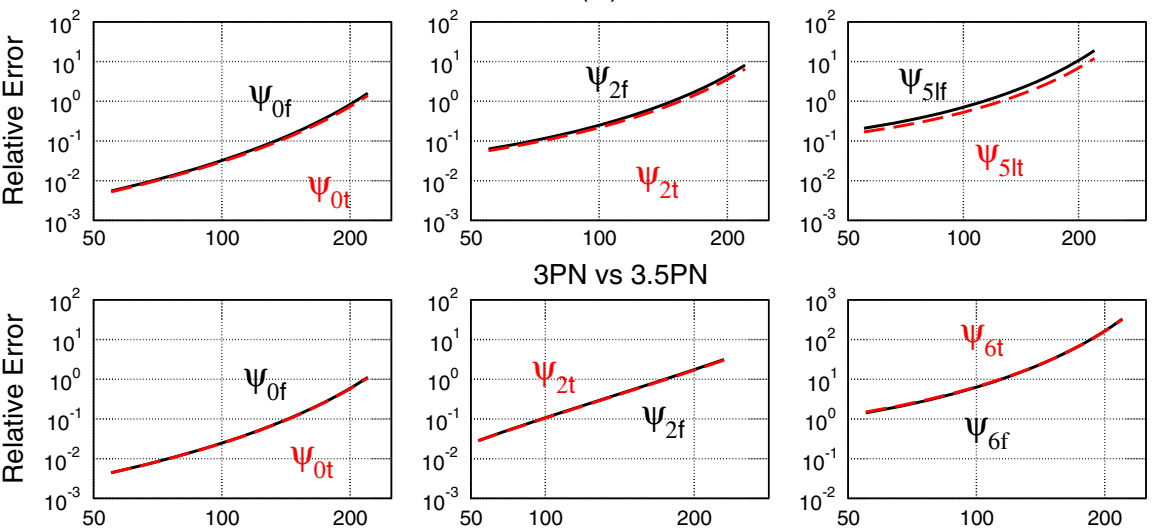

3PN vs 3.5PN
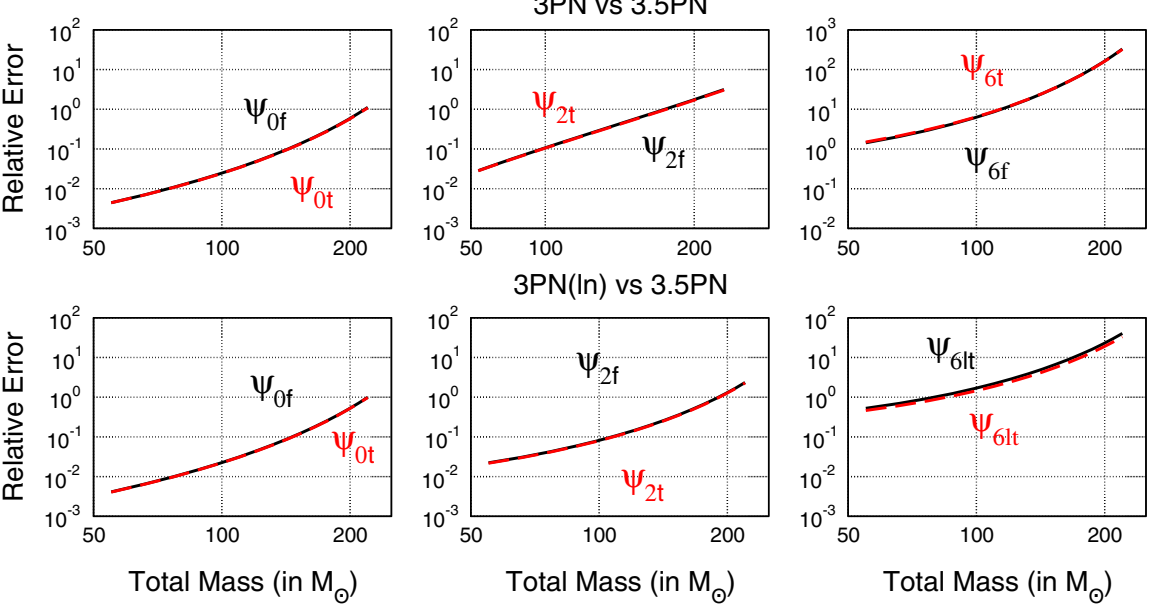

FIG. 9 (color online). A comparison of relative errors in the measurement of various PN parameters for binaries with masses in the range $55-220 M_{\odot}$ at a luminosity distance of $3 \mathrm{Gpc}$ for two cases: The first is the same as before when $\psi_{0}$ and $\psi_{2}$ are basic parameters and all PN parameters up to 3.5PN (full phasing) except the test parameter are parametrized by $\psi_{0}$ and $\psi_{2}$. The others are similarly constructed but the phasing is truncated at the PN order corresponding to the test parameter. The low-frequency cutoff is $10 \mathrm{~Hz}$ and the RWF has been used. The test parameter with truncated phasing is denoted by $\psi_{i t}$ while with full $3.5 \mathrm{PN}$ phasing it is denoted by $\psi_{i f}$.

a basic parameter than when it is a test parameter. This is mainly because the basic variables bring new functional dependences via the rest of the phasing terms.

(2) The choice of the lower-order PN coefficient $\psi_{2}$ as a basic variable leads to a more precise test.

(3) When $\psi_{0}$ is one of the basic variables, the dispersion in the relative error of the other basic variable is least when the lowest-order PN coefficient $\psi_{2}$ is chosen as the second basic variable.
(4) An interesting case corresponds to the choice of $\psi_{4}$ as the basic variable which seems to allow for the best determination of $\psi_{4}$.

As a result, although, in principle, one has the freedom of parametrizing the waveform in terms of any of the two PN coefficients, the choice of $\left(\psi_{0}, \psi_{2}\right)$ as basic variables is the optimal one.

The above question may be equivalently investigated by looking at the volume of the three-dimensional ellipsoid 
TABLE I. Accuracies of the measurement of various PN parameters (using the RWF and $1 \mathrm{~Hz}$ low-frequency cutoff) for $(10,100) M_{\odot}$ binary located at a luminosity distance of $3 \mathrm{Gpc}$, with different choices of parametrization schemes. For each entry, the number within parentheses is the factor by which the accuracy will be reduced if a lower cutoff of $10 \mathrm{~Hz}$ is chosen instead of $1 \mathrm{~Hz}$. For the fundamental pair we have chosen $\left(\psi_{0}, \psi_{f}\right)$, where $f$ can be any of $2,3,4,5 l, 6,6 l$, or 7 . In each case, the relative error in the test parameter is listed in the third row.

\begin{tabular}{|c|c|c|c|c|c|c|c|}
\hline \multicolumn{8}{|c|}{ 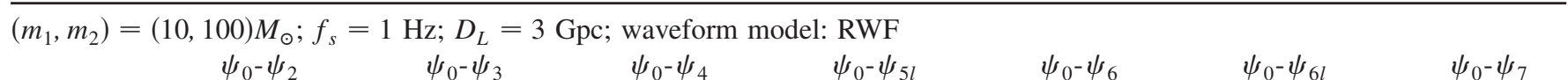 } \\
\hline$\Delta \psi_{0} / \psi_{0}$ & $\ldots$ & $0.0015(60)$ & $0.0015(60)$ & $0.0015(60)$ & $0.0015(60)$ & $0.0015(60)$ & $0.0015(60)$ \\
\hline$\Delta \psi_{f} / \psi_{f}$ & $\ldots$ & $0.0092(15)$ & $0.010(17)$ & $0.017(18)$ & $0.043(17)$ & 0.020 (19) & $0.022(19)$ \\
\hline$\Delta \psi_{2} / \psi_{2}$ & $\cdots$ & 0.027 & 0.027 (27) & 0.027 (27) & 0.027 (27) & 0.027 (27) & 0.027 (27) \\
\hline$\Delta \psi_{0} / \psi_{0}$ & $0.0010(55)$ & $\ldots$ & $0.0010(55)$ & $0.0010(55)$ & $0.0010(55)$ & $0.0010(55)$ & $0.0010(55)$ \\
\hline$\Delta \psi_{f} / \psi_{f}$ & 0.0089 (13) & $\ldots$ & $0.020(16)$ & $0.031(16)$ & $0.082(16)$ & $0.037(16)$ & $0.042(16)$ \\
\hline$\Delta \psi_{3} / \psi_{3}$ & $0.0050(42)$ & $\cdots$ & $0.0050(42)$ & $0.0050(42)$ & $0.0050(42)$ & $0.0050(42)$ & $0.0050(42)$ \\
\hline$\Delta \psi_{0} / \psi_{0}$ & $0.0011(28)$ & $0.0011(28)$ & $\cdots$ & $0.0011(28)$ & $0.0011(28)$ & $0.0011(28)$ & $0.0011(28)$ \\
\hline$\Delta \psi_{f} / \psi_{f}$ & $0.074(8)$ & $0.15(8)$ & $\ldots$ & $0.25(8)$ & $0.65(8)$ & $0.29(8)$ & $0.33(8)$ \\
\hline$\Delta \psi_{4} / \psi_{4}$ & $2.1(8)$ & $2.1(8)$ & $\cdots$ & $2.1(8)$ & $2.1(8)$ & $2.1(8)$ & $2.1(8)$ \\
\hline$\Delta \psi_{0} / \psi_{0}$ & 0.00059 (77) & $0.00059(77)$ & $0.00059(77)$ & $\ldots$ & $0.00059(77)$ & $0.00059(77)$ & $0.00059(77)$ \\
\hline$\Delta \psi_{f} / \psi_{f}$ & $0.014(24)$ & $0.026(23)$ & $0.029(23)$ & $\ldots$ & $0.12(23)$ & $0.052(23)$ & $0.058(23)$ \\
\hline$\Delta \psi_{5 l} / \psi_{5 l}$ & $0.056(17)$ & $0.056(17)$ & 0.056 (17) & $\cdots$ & $0.056(17)$ & $0.056(17)$ & 0.056 (17) \\
\hline$\Delta \psi_{0} / \psi_{0}$ & $0.00054(64)$ & $0.00054(64)$ & $0.00054(64)$ & $0.00054(64)$ & $\cdots$ & $0.00054(64)$ & $0.00054(64)$ \\
\hline$\Delta \psi_{f} / \psi_{f}$ & $0.0067(21)$ & $0.013(20)$ & 0.014 (19) & 0.021 (19) & $\cdots$ & 0.025 (19) & 0.028 (19) \\
\hline$\Delta \psi_{6} / \psi_{6}$ & 0.67 (13) & 0.67 (13) & $0.67(13)$ & 0.67 (13) & $\ldots$ & $0.67(13)$ & $0.67(13)$ \\
\hline$\Delta \psi_{0} / \psi_{0}$ & $0.00051(62)$ & $0.00051(62)$ & $0.00051(62)$ & $0.00051(62)$ & $0.00051(62)$ & $\ldots$ & $0.00051(62)$ \\
\hline$\Delta \psi_{f} / \psi_{f}$ & $0.0051(21)$ & $0.0096(19)$ & 0.010 (19) & 0.016 (19) & 0.042 (19) & $\cdots$ & $0.021(18)$ \\
\hline$\Delta \psi_{6 l} / \psi_{6 l}$ & $0.17(13)$ & $0.17(13)$ & 0.17 (13) & 0.17 (13) & $0.17(13)$ & $\cdots$ & $0.17(13)$ \\
\hline$\Delta \psi_{0} / \psi_{0}$ & 0.00049 (59) & 0.00049 (59) & 0.00049 (59) & 0.00049 (59) & 0.00049 (59) & $0.00049(59)$ & $\cdots$ \\
\hline$\Delta \psi_{f} / \psi_{f}$ & $0.0046(20)$ & 0.0087 (18) & $0.0094(18)$ & 0.014 (17) & $0.038(18)$ & 0.017 (17) & $\cdots$ \\
\hline$\Delta \psi_{7} / \psi_{7}$ & 0.19 (10) & $0.19(10)$ & $0.19(10)$ & $0.19(10)$ & $0.19(10)$ & $0.19(10)$ & $\cdots$ \\
\hline
\end{tabular}

corresponding to the three phasing coefficients which are involved in the test. We find that the smallest volume of the ellipsoid corresponds to the case where $\psi_{0}$ and $\psi_{2}$ are used as basic variables as compared to other combinations, for

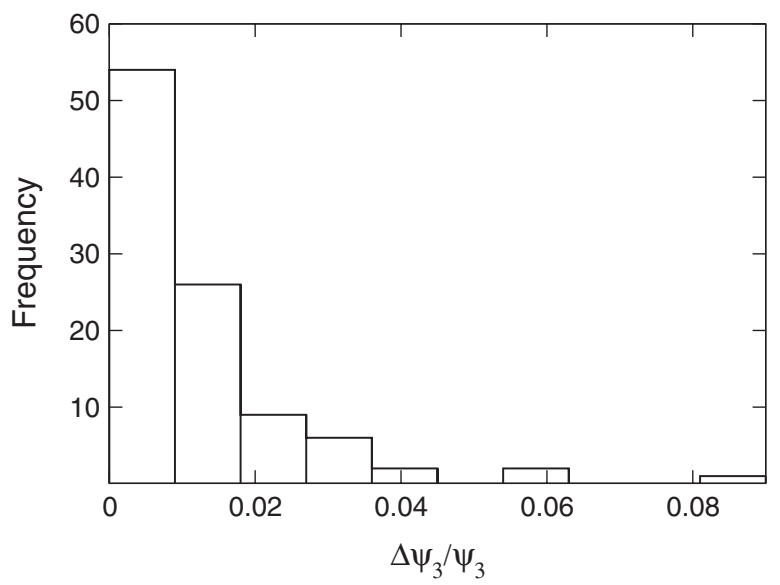

FIG. 10. Histogram for the relative error in the estimation of the parameter $\psi_{3}$ using 100 different realizations of angular parameters for a $(10,100) M_{\odot}$ binary located at the luminosity distance of $3 \mathrm{Gpc}$. The low-frequency cutoff is $1 \mathrm{~Hz}$ and RWF has been used. all test parameters except $\psi_{4}$ and $\psi_{6}$. For these two parameters the volume is smaller when they are used as basic variables together with $\psi_{0}$.

\section{The choice of angles}

In Sec. II we pointed out that the signal depends on four angular parameters $(\cos \theta, \phi, \psi, \cos \iota)$ related to the source location and orientation but that they were chosen arbitrarily to be $\theta=\phi=\pi / 6, \psi=\pi / 4$, and $\iota=\pi / 3$ in the present study. This is because for terrestrial detectors and burst sources the angles could be considered constant. To quantify the effect of these angular dependences on the test we computed the relative error in a particular PN parameter for 100 different realizations of these angular parameters.

The result is plotted in Fig. 10. From Figs. 7 and 10, it is clear that the value of the relative error in the estimation of $\psi_{3}$ for a $(10,100) M_{\odot}$ binary located at a luminosity distance of $3 \mathrm{Gpc}$ is a typical value and, as was physically expected, the weak dependence on angles is a good approximation.

\section{SUMMARY AND CONCLUDING REMARKS}

In this paper we have studied the possibility of testing the theory of gravity within a well-defined subclass of $\mathrm{ppE}$ 
theories using $\mathrm{GW}$ observations of $\mathrm{BBHs}$ by a typical second generation GW interferometer (Advanced LIGO) and the plausible third generation GW interferometer (ET). Within this subclass of theories for Advanced LIGO we have shown that $\mathrm{GW}$ observations of BBHs (in the range $11-110 M_{\odot}$ and at a luminosity distance of $300 \mathrm{Mpc}$ ) can be used to estimate only the PN coefficient $\psi_{3}$ with fractional accuracy better than $6 \%$ when the FWF is used (see Fig. 4). Estimation of a PN coefficient with such an accuracy suggests that Advanced LIGO could indeed begin the era of strong-field tests of gravity. We have also compared the results for the FWF and RWF and shown that FWF reduces the errors by a factor of 3 to almost 100 .

We have also studied in detail the stellar mass and intermediate mass regimes of the compact binary source population in the ET sensitivity band, for 1 and $10 \mathrm{~Hz}$ lower cutoff frequencies and compared the advantage of using the FWF model over the RWF model. We find that the lower frequency cutoff of $1 \mathrm{~Hz}$ plays a crucial role in testing GR with ET.

For stellar mass binary coalescences (total mass $\leq 44 M_{\odot}$ ) as well as intermediate $\mathrm{BH}$ binaries, the lower cutoff of $1 \mathrm{~Hz}$ improves the estimation of all PN parameters in the phasing formula. For stellar mass binaries, the improvement in the estimation is between a factor of 2 to almost 20 when the RWF model is used. When the FWF model is used, the improvements are typically between factors 2-10 (see Fig. 5).

For intermediate mass binaries, which coalesce at lower frequencies, though the smaller lower cutoff improves the parameter estimation, the errors associated with the measurement of various parameters are so large that the tests are not very interesting (see Fig. 7). However, when total mass is less than about $100 M_{\odot}$, all the $\psi_{k}$ 's are measured with relative errors less than unity, the most accurately determined parameters being $\psi_{3}$ and $\psi_{5 l}$, which are determined with accuracies better than $10 \%$. This seems to be the most interesting mass range for the proposed test in the ET band. Though the use of the FWF does improve the estimation of various parameters, the test is less impressive since for astrophysically realistic event rates, we have to consider distances as large as $3 \mathrm{Gpc}$ (as opposed to $300 \mathrm{Mpc}$ for the stellar mass case). Thus, only if there is such an event very close by, can the test be performed very accurately.

It is worth bearing in mind that in addition to systematic effects due to higher-order PN terms, various other systematic effects could offset the accuracy of the proposed test:

(1) If the components of the binaries have spins, the phasing coefficients are functions not only of the individual masses but also the spin parameters. Further, if the binary is precessing (which would be the case when the spins are not aligned or antialigned with the orbital angular momentum vector of the binary), the waveforms will have a very different structure due to spin-induced modulations. To get a simple estimate of the effect of spins on the proposed test, we consider spinning but nonprecessing binaries. For such binaries, the effect of spin is to introduce additional spin-dependent contributions in various phasing coefficients at and above $1.5 \mathrm{PN}$. The 1.5PN phasing coefficient in this case has an additional spin parameter $\beta$, which is a function of the individual spins of the binary and takes values $0 \leq \beta \leq 8.5$ [52]. We found that for values of $\beta \geq 6$, the bias in our estimate could be more than $100 \%$. This means that the presence of spins could significantly bias the proposed test for large values of the spin parameter.

(2) Another effect is that of the orbital eccentricity, which we have ignored by assuming the binary's orbit to be quasicircular. As shown in Refs. [16,53], orbital eccentricity will introduce additional phasing coefficients with completely different frequency dependences. It will need a careful study to assess how to incorporate the effect of eccentricity into our analysis, which we postpone to a future work.

(3) Lastly, since we have used PN inspiral waveforms, the neglect of merger and ringdown effects could also lead to further systematic errors. By a proper choice of the domain of integration of the signal, we should be able to take care of it to some extent. A detailed study using some of the analytic parametrizations of numerical relativity waveforms (see Refs. [54-56]) is planned as a followup of this work.

In this paper we have explored issues and indicated ways to test a class of theories of gravitation close to GR by using GW observations in Advanced LIGO and the Einstein Telescope. The extension of these results within a more general class of models like the ppE framework can be expected to provide more general results in the future.

To fully test our proposal one must mimic the whole exercise with mock data. One has to inject a non-GR signal into Gaussian background with a signal that differs from GR at 1.5PN and higher orders by a certain degree. One would then need to extract, say, the first three parameters by a Markov chain Monte Carlo technique that employs GR templates, and see if what we expect based on our toy examples above holds good. We are currently exploring this exercise but this exercise is quite compute intensive and goes beyond the scope of the present paper and so we defer its full discussion to a future publication.

\section{ACKNOWLEDGMENTS}

We thank Collin Capano (Syracuse) for providing the analytical fit for the Advanced LIGO sensitivity curve. K. G. A. thanks Clifford Will for discussions. K. G. A. 
acknowledges support by the National Science Foundation, Grant No. PHY 06-52448, the National Aeronautics and Space Administration, Grant No. NNG-06GI60G, and the Centre National de la Recherche Scientifique, Programme International de Coopération Scientifique (CNRS-PICS), Grant No. 4396. B. S. S. was supported in part by PPARC Grant No. PP/B500731/1.

\section{APPENDIX: SYSTEMATIC EFFECT DUE TO SPIN}

We discuss the typical biases on our estimates due to the assumption that the binary components are nonspinning. We demonstrate this, by taking the $1.5 \mathrm{PN}$ phasing coefficient, where the spins first enter the phasing. For convenience, we have assumed the spins of the binary are aligned with the orbital angular momentum vector, in which case we can use the direct analytical formula for the phasing coefficient.

As we mentioned earlier, the nonspinning $1.5 \mathrm{PN}$ phasing coefficient is given by $\alpha_{3}^{\text {nonspin }}=-16 \pi$. The corresponding expression for spinning but nonprecessing binaries is $\alpha_{3}^{\text {spin }}=-16 \pi+4 \beta$, where $\beta$ is a spin parameter which is a function of the spins of the individual components of the binary and whose value lies in the range $0 \leq \beta \leq 8.5$ [52]. Thus the difference in the value of the $1.5 \mathrm{PN}$ coefficient due to spin is $\delta \alpha_{3}^{\text {spin }}=\alpha_{3}^{\text {spin }}-\alpha_{3}^{\text {nonspin }}=4 \beta$. The bias in our estimates of $\frac{\Delta \alpha_{3}}{\alpha_{3}^{\text {nonspin }}}$ is given by

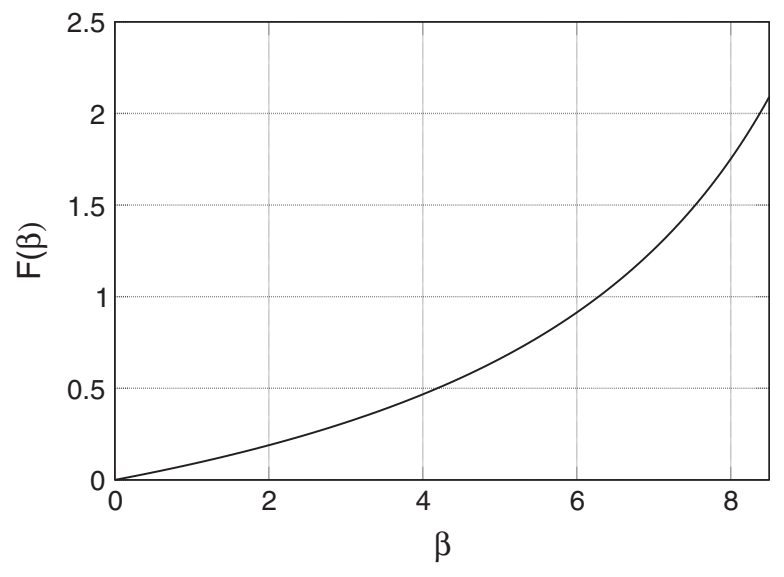

FIG. 11. This plot shows the variation of systematic bias due to spin $F(\beta)$ with the spin parameter $0 \leq \beta \leq 8.5$, where $F(\beta)$ is given by $F(\beta)=4 \beta(16 \pi-4 \beta)^{-1}$.

$$
\frac{\Delta \alpha_{3}}{\alpha_{3}^{\text {spin }}}-\frac{\Delta \alpha_{3}}{\alpha_{3}^{\text {nonspin }}}=\frac{\Delta \alpha_{3}}{\alpha_{3}^{\text {nonspin }}} \times F(\beta),
$$

where $F(\beta)=\frac{4 \beta}{(16 \pi-4 \beta)}$ quantifies the bias in our estimate.

Figure11 shows the plot of $F(\beta)$. As is obvious, the systematic bias due to spins could offset the estimation of $\alpha_{3}$ by more than $100 \%$ for $\beta \geq 6$.
[1] C. M. Will, Living Rev. Relativity 9, 3 (2006), http:// relativity.livingreviews.org/Articles/lrr-2006-3/.

[2] C.M. Will, Theory and Experiments in Gravitational Physics (Cambridge University Press, New York, 1981).

[3] T. Damour and J.H. Taylor, Phys. Rev. D 45, 1840 (1992).

[4] T. Damour and N. Deruelle, Ann. Inst. Henri Poincaré Phys. Théor. 43, 107 (1985).

[5] T. Damour and N. Deruelle, Ann. Inst. Henri Poincaré Phys. Théor. 44, 263 (1986).

[6] J. Taylor and J. Weisberg, Astrophys. J. 253, 908 (1982).

[7] B.S. Sathyaprakash and B.F. Schutz, Living Rev. Relativity 12, 2 (2009), http://relativity.livingreviews.org/ Articles/lrr-2009-2/.

[8] A. Buonanno, B. R. Iyer, E. Ochsner, Y. Pan, and B.S. Sathyaprakash, Phys. Rev. D 80, 084043 (2009).

[9] http://www.ligo.caltech.edu.

[10] http://www.virgo.infn.it.

[11] L. Blanchet and B.S. Sathyaprakash, Classical Quantum Gravity 11, 2807 (1994).

[12] L. Blanchet and B. S. Sathyaprakash, Phys. Rev. Lett. 74, 1067 (1995).

[13] F. D. Ryan, Phys. Rev. D 56, 1845 (1997).

[14] C. M. Will, Phys. Rev. D 50, 6058 (1994).

[15] C. M. Will, Phys. Rev. D 57, 2061 (1998).
[16] A. Królak, K. D. Kokkotas, and G. Schäfer, Phys. Rev. D 52, 2089 (1995).

[17] C. M. Will and N. Yunes, Classical Quantum Gravity 21, 4367 (2004).

[18] E. Berti, A. Buonanno, and C. M. Will, Phys. Rev. D 71, 084025 (2005).

[19] K. G. Arun and C. M. Will, Classical Quantum Gravity 26, 155002 (2009).

[20] A. Stavridis and C. M. Will, Phys. Rev. D 80, 044002 (2009).

[21] K. Yagi and T. Tanaka, Phys. Rev. D 81, 064008 (2010).

[22] L. S. Finn and P. J. Sutton, Phys. Rev. D 65, 044022 (2002).

[23] K. G. Arun, B. R. Iyer, M.S.S. Qusailah, and B.S. Sathyaprakash, Classical Quantum Gravity 23, L37 (2006).

[24] M.S.S. Qusailah, Ph.D. thesis, Jawaharlal Nehru University, New Delhi, 2006.

[25] K. G. Arun, B. R. Iyer, M.S.S. Qusailah, and B.S. Sathyaprakash, Phys. Rev. D 74, 024006 (2006).

[26] O. Dreyer, B. Kelly, B. Krishnan, L. S. Finn, D. Garrison, and R. Lopez-Aleman, Classical Quantum Gravity 21, 787 (2004).

[27] E. Berti, V. Cardoso, and C. M. Will, Phys. Rev. D 73, 064030 (2006). 
[28] S. A. Hughes and K. Menou, Astrophys. J. 623, 689 (2005).

[29] D. Keppel and P. Ajith, arXiv:1004.0284.

[30] S. Alexander, L. S. Finn, and N. Yunes, Phys. Rev. D 78, 066005 (2008).

[31] C. Molina, P. Pani, V. Cardoso, and L. Gualtieri, Phys. Rev. D 81, 124021 (2010).

[32] N. Yunes and F. Pretorius, Phys. Rev. D 80, 122003 (2009).

[33] U. Cannella, S. Foffa, M. Maggiore, H. Sanctuary, and R. Sturani, Phys. Rev. D 80, 124035 (2009).

[34] C.F. Sopuerta and N. Yunes, Phys. Rev. D 80, 064006 (2009).

[35] I. Mandel, J. R. Gair, and M. C. Miller, arXiv:0912.4925.

[36] https://dcc.ligo.org/cgi-bin/DocDB/ ShowDocument?docid $=2974$.

[37] S. Hild, S. Chelkowski, and A. Freise, arXiv:0810.0604.

[38] L. Blanchet, G. Faye, B. R. Iyer, and S. Sinha, Classical Quantum Gravity 25, 165003 (2008).

[39] L. Blanchet, G. Faye, B. R. Iyer, and B. Joguet, Phys. Rev. D 65, 061501(R) (2002); 71, 129902(E) (2005).

[40] L. Blanchet, T. Damour, G. Esposito-Farèse, and B. R. Iyer, Phys. Rev. Lett. 93, 091101 (2004).

[41] K. G. Arun, B. R. Iyer, B.S. Sathyaprakash, S. Sinha, and C. Van Den Broeck, Phys. Rev. D 76, 104016 (2007).

[42] C. Van Den Broeck and A.S. Sengupta, Classical Quantum Gravity 24, 1089 (2007).
[43] L. S. Finn, Phys. Rev. D 46, 5236 (1992).

[44] L. S. Finn and D.F. Chernoff, Phys. Rev. D 47, 2198 (1993).

[45] C. Helström, in Statistical Theory of Signal Detection, International Series of Monographs in Electronics and Instrumentation Vol. 9 (Pergamon Press, Oxford, UK, 1968), 2nd ed.

[46] L.A. Wainstein and V.D. Zubakov, Extraction of Signals from Noise (Prentice-Hall, Englewood Cliffs, NJ, 1962).

[47] C. Van Den Broeck and A. Sengupta, Classical Quantum Gravity 24, 155 (2007).

[48] L.P. Grishchuk, V. M. Lipunov, K. A. Postnov, M.E. Prokhorov, and B.S. Sathyaprakash, Usp. Fiz. Nauk 171, 3 (2001) [Phys. Usp. 44, 1 (2001)].

[49] J. Abadie et al. (LIGO Scientific Collaboration), Classical Quantum Gravity 27, 173001 (2010).

[50] I. Mandel, D. A. Brown, J.R. Gair, and M. C. Miller, Astrophys. J. 681, 1431 (2008).

[51] A. Sesana, J. Gair, I. Mandel, and A. Vecchio, Astrophys. J. 698, L129 (2009).

[52] C. Cutler and E.E. Flanagan, Phys. Rev. D 49, 2658 (1994).

[53] N. Yunes, K. G. Arun, E. Berti, and C. M. Will, Phys. Rev. D 80, 084001 (2009).

[54] P. Ajith et al., Phys. Rev. D 77, 104017 (2008).

[55] Y. Pan et al., Phys. Rev. D 81, 084041 (2010).

[56] T. Damour and A. Nagar, Phys. Rev. D 81, 084016 (2010). 